\title{
The squashed entanglement of a quantum channel
}

\author{
Masahiro Takeoka*† Saikat Guha ${ }^{\dagger} \quad$ Mark M. Wilde ${ }^{\ddagger}$
}

January 22, 2014

\begin{abstract}
This paper defines the squashed entanglement of a quantum channel as the maximum squashed entanglement that can be registered by a sender and receiver at the input and output of a quantum channel, respectively. A new subadditivity inequality for the original squashed entanglement measure of Christandl and Winter leads to the conclusion that the squashed entanglement of a quantum channel is an additive function of a tensor product of any two quantum channels. More importantly, this new subadditivity inequality, along with prior results of Christandl, Winter, et al., establishes the squashed entanglement of a quantum channel as an upper bound on the quantum communication capacity of any channel assisted by unlimited forward and backward classical communication. A similar proof establishes this quantity as an upper bound on the private capacity of a quantum channel assisted by unlimited forward and backward public classical communication. This latter result is relevant as a limitation on rates achievable in quantum key distribution. As an important application, we determine that these capacities can never exceed $\log ((1+\eta) /(1-\eta))$ for a pure-loss bosonic channel for which a fraction $\eta$ of the input photons make it to the output on average. The best known lower bound on these capacities is equal to $\log (1 /(1-\eta))$. Thus, in the high-loss regime for which $\eta \ll 1$, this new upper bound demonstrates that the protocols corresponding to the above lower bound are nearly optimal.
\end{abstract}

\section{Introduction}

One of the seminal insights of classical information theory is that public discussion between two parties trying to communicate privately can enhance their ability to do so [Mau93, AC93. Indeed, let $p_{Y, Z \mid X}(y, z \mid x)$ be a stochastic map modeling a broadcast channel that connects a sender $X$ to a legitimate receiver $Y$ and a wiretapper $Z$. Maurer [Mau93] and Ahslwede and Csiszár [AC93] independently discovered that the so-called secret-key agreement capacity of such a broadcast channel $]_{1}^{1}$ in which public discussion is allowed, can be strictly larger than zero even if the private capacity of the channel is equal to zero (the capacity for private communication without any public discussion). This result has shaped the formulation of practical protocols for secret key agreement.

\footnotetext{
${ }^{*}$ National Institute of Information and Communications Technology, Koganei, Tokyo 184-8795, Japan

${ }^{\dagger}$ Quantum Information Processing Group, Raytheon BBN Technologies, Cambridge, MA 02138, USA

${ }^{\ddagger}$ Hearne Institute for Theoretical Physics, Department of Physics and Astronomy, Center for Computation and Technology, Louisiana State University, Baton Rouge, Louisiana 70803, USA

${ }^{1}$ Note that the secret-key agreement capacity is equal to the capacity for private communication with unlimited public discussion, due to the one-time pad protocol.
} 
In later work, Maurer and Wolf introduced the intrinsic information and proved that it is a sharp upper bound on the secret key agreement capacity [MW99]. It is defined as follows:

$$
\max _{p_{X}(x)} I(X ; Y \downarrow Z)
$$

where $I(X ; Y \downarrow Z)$ is equal to a minimization of the conditional mutual information over all stochastic maps with which an adversary possessing $Z$ can act to produce $\bar{Z}$ :

$$
I(X ; Y \downarrow Z) \equiv \min _{p_{\bar{Z} \mid Z}(\bar{z} \mid z)} I(X ; Y \mid \bar{Z}) .
$$

The conditional mutual information is defined as

$$
I(X ; Y \mid \bar{Z}) \equiv H(X \bar{Z})+H(Y \bar{Z})-H(X Y \bar{Z})-H(\bar{Z})
$$

where the Shannon entropies are evaluated with respect to the marginal distributions resulting from the joint distribution $p_{X}(x) p_{Y, Z \mid X}(y, z \mid x) p_{\bar{Z} \mid Z}(\bar{z} \mid z)$. The interpretation of the intrinsic information is that it is a measure of the correlations that the sender and legitimate receiver can establish, with the adversary acting in the strongest possible way to reduce these correlations.

Due to strong parallels discovered between secrecy and quantum coherence or entanglement [SW98, LC99, SP00], Christandl realized that an extension of the intrinsic information to quantum information theory might be helpful in simplifying the arduous task of quantifying entanglement present in quantum states [Chr02]. This realization then culminated in the establishment of the squashed entanglement $E_{\mathrm{sq}}(A ; B)_{\rho}$ of a bipartite quantum state $\rho_{A B}$ as an upper bound on the rate at which two parties can distill Bell states $(|0\rangle|0\rangle+|1\rangle|1\rangle) / \sqrt{2}$ from many copies of $\rho_{A B}$ by performing local operations and classical communication [CW04. A similar proof technique establishes squashed entanglement as an upper bound on distillable secret key [CEH ${ }^{+}$], and this approach has the benefit of being conceptually simpler than the original classical approaches from [Mau93, AC93. The squashed entanglement $E_{\mathrm{sq}}(A ; B)_{\rho}$ is defined as the following function of a bipartite state $\rho_{A B}$ :

$$
E_{\mathrm{sq}}(A ; B)_{\rho} \equiv \frac{1}{2} \inf _{\mathcal{S}_{E \rightarrow E^{\prime}}} I\left(A ; B \mid E^{\prime}\right),
$$

where the conditional quantum mutual information is defined similarly to the classical one (however with Shannon entropies replaced by von Neumann entropies) and the infimum is with respect to all noisy "squashing channels" $\mathcal{S}_{E \rightarrow E^{\prime}}$ taking the $E$ system of a purification $\left|\phi^{\rho}\right\rangle_{A B E}$ of $\rho_{A B}$ to a system $E^{\prime}$ of arbitrary dimension. In related work, Tucci has defined a functional bearing some similarities to squashed entanglement [Tuc99, Tuc02].

The similarities between (3) and (1) are evident and the interpretations are similar. That is, we interpret $E_{\mathrm{sq}}(A ; B)_{\rho}$ as quantifying the quantum correlations between $A$ and $B$ after an adversary possessing the purifying system $E$ performs a quantum channel with the intent of "squashing down" the correlations that they share. Due to a lack of an upper bound on the dimension of the output $E^{\prime}$ of the squashing channel, it is not yet known whether the infimization in (3) can be replaced by a minimization, but note that such a replacement is possible for the classical intrinsic information [CRW03. Furthermore, it is not even clear that, given a description of a density matrix $\rho_{A B}$, the computation of its squashed entanglement can be performed in non-deterministic polynomial time (NP). However, these apparent difficulties are not an obstruction to finding good upper bounds on the distillable entanglement or distillable secret key of a quantum state $\rho_{A B}$ : the results of [CW04, 
$\mathrm{CEH}^{+} 07$ ] are that any squashing channel gives an upper bound on these quantities and so the task is to find the best one in a reasonable amount of time. Finally, among the many entanglement measures, squashed entanglement is the only one known to satisfy all eight desirable properties that have arisen in the axiomatization of entanglement theory [CW04, KW04, AF04, BCY11].

\section{Summary of results}

In this paper, we provide the following contributions:

1. Our first contribution is to define the squashed entanglement of a quantum channel $\mathcal{N}_{A^{\prime} \rightarrow B}$ as the maximum squashed entanglement that can be registered between a sender and receiver who have access to the input $A^{\prime}$ and output $B$ of this channel, respectively:

$$
E_{\mathrm{sq}}(\mathcal{N}) \equiv \max _{|\phi\rangle_{A A^{\prime}}} E_{\mathrm{sq}}(A ; B)_{\rho},
$$

where $\rho_{A B} \equiv \mathcal{N}_{A^{\prime} \rightarrow B}\left(|\phi\rangle\left\langle\left.\phi\right|_{A A^{\prime}}\right)\right.$. The formula above is formally analogous to the classical formula in $(1)$, and the rest of this paper establishes that $E_{\mathrm{sq}}(\mathcal{N})$ plays an analogous operational role in the quantum setting.

2. One of the main technical contributions of this paper is a proof of a new subadditivity inequality (Theorem 7) for the squashed entanglement.

3. This inequality has two important implications. First, Theorem 7 implies that $E_{\mathrm{sq}}(\mathcal{N})$ is additive as a function of channels, in the sense that $E_{\mathrm{sq}}(\mathcal{N} \otimes \mathcal{M})=E_{\mathrm{sq}}(\mathcal{N})+E_{\mathrm{sq}}(\mathcal{M})$ for any two channels $\mathcal{N}$ and $\mathcal{M}$. Thus, the squashed entanglement of a channel is a well behaved function of channels.

4. Next, and more importantly, Theorem 7 is helpful in establishing $E_{\mathrm{sq}}(\mathcal{N})$ as an upper bound on the quantum communication capacity of a channel $\mathcal{N}$ assisted by unlimited forward and backward classical communication (hereafter denoted as $Q_{2}(\mathcal{N})$ ). This new squashed entanglement upper bound is an improvement upon the best previously known upper bound on $Q_{2}(\mathcal{N})$ given in terms of the entanglement cost of a quantum channel [BBCW13, with the improvement following from the fact that the squashed entanglement is never larger than the entanglement of formation [CW04. In addition to being tighter, our bound is "single-letter," meaning that it can be evaluated as a function of a single channel use, whereas the bound from BBCW13] is regularized, meaning that it is intractable to evaluate it in spite of being able to write down a formal mathematical expression for it. By a similar proof, we find that $E_{\mathrm{sq}}(\mathcal{N})$ is a single-letter upper bound on the private capacity of a channel $\mathcal{N}$ assisted by unlimited forward and backward public classical communication (hereafter denoted as $P_{2}(\mathcal{N})$ ). These latter results represent important progress on one of the longest standing open questions in quantum information theory [BDS97] (namely, to determine these capacities or sharpen the bounds on them).

5. As examples, we compute upper bounds on $Q_{2}$ and $P_{2}$ for all qubit Pauli channels, and we consider the special cases of a qubit dephasing channel and a qubit depolarizing channel. 
6. Finally, we show that our upper bound on $Q_{2}\left(\mathcal{N}_{\eta}\right)$ and $P_{2}\left(\mathcal{N}_{\eta}\right)$ for the pure-loss bosonic channel $\mathcal{N}_{\eta}$ with transimissivity $\eta \in[0,1]$, is very close to the best-known lower bound on these capacities from [GPLS09, PGBL09, in the practically-relevant regime of high loss $(\eta \ll 1)$. This result puts an upper limit on the secret-key rate achievable by any optical quantum key distribution protocol. We also establish an upper bound on $Q_{2}$ and $P_{2}$ for all phase-insensitive Gaussian channels, which includes the thermal and additive noise Gaussian channels.

\section{Properties of the squashed entanglement of a quantum channel}

We begin with our main definition:

Definition 1 The squashed entanglement of a quantum channel $\mathcal{N}_{A^{\prime} \rightarrow B}$ is the maximum squashed entanglement that can be registered between a sender and receiver who have access to the input $A^{\prime}$ and output $B$ of this channel, respectively:

$$
E_{\mathrm{sq}}(\mathcal{N}) \equiv \max _{|\phi\rangle_{A A^{\prime}}} E_{\mathrm{sq}}(A ; B)_{\rho}
$$

where $\rho_{A B} \equiv \mathcal{N}_{A^{\prime} \rightarrow B}\left(|\phi\rangle\left\langle\left.\phi\right|_{A A^{\prime}}\right)\right.$.

Remark 2 We can restrict the optimization in (4) to be taken over pure bipartite states rather than mixed ones, due to the convexity of squashed entanglement (see Proposition 3 of [CW04]). In more detail, let $\sigma_{A A^{\prime}}$ be a mixed state on systems $A$ and $A^{\prime}$. Then it has a spectral decomposition of the following form:

$$
\sigma_{A A^{\prime}}=\sum_{x} p_{X}(x)\left|\psi_{x}\right\rangle\left\langle\left.\psi_{x}\right|_{A A^{\prime}}\right.
$$

Let $\omega_{A B} \equiv \mathcal{N}_{A^{\prime} \rightarrow B}\left(\sigma_{A A^{\prime}}\right)$ and $\omega_{A B}^{x} \equiv \mathcal{N}_{A^{\prime} \rightarrow B}\left(\left|\psi_{x}\right\rangle\left\langle\left.\psi_{x}\right|_{A A^{\prime}}\right)\right.$. Then the following inequality holds, due to convexity of the squashed entanglement:

$$
E_{\mathrm{sq}}(A ; B)_{\omega} \leq \sum_{x} p_{X}(x) E_{\mathrm{sq}}(A ; B)_{\omega^{x}}
$$

From this, we conclude that for any mixed input state $\sigma_{A A^{\prime}}$, the following inequality holds

$$
E_{\mathrm{sq}}(A ; B)_{\omega} \leq \max _{|\phi\rangle_{A A^{\prime}}} E_{\mathrm{sq}}(A ; B)_{\rho}
$$

where $\rho_{A B} \equiv \mathcal{N}_{A^{\prime} \rightarrow B}\left(|\phi\rangle\left\langle\left.\phi\right|_{A A^{\prime}}\right)\right.$, so that it suffices to optimize over pure bipartite input states.

Remark 3 Note that we can indeed take a maximization (rather than a supremization) over pure bipartite inputs if the input space is finite-dimensional because in this case, the input space is compact and the squashed entanglement measure is continuous [AF04].

Lemma 4 We can alternatively write the squashed entanglement of a quantum channel as

$$
\frac{1}{2} \max _{\rho_{A^{\prime}}} \inf _{V_{E \rightarrow E^{\prime} F}}\left[H\left(B \mid E^{\prime}\right)_{\omega}+H(B \mid F)_{\omega}\right],
$$


where the maximization is over density operators $\rho_{A^{\prime}}$ on the input system $A^{\prime}$, the infimization is over "squashing isometries" $V_{E \rightarrow E^{\prime} F}$, and the entropies are with respect to the state $\omega_{B E^{\prime} F}$, defined as

$$
\omega_{B E^{\prime} F} \equiv V_{E \rightarrow E^{\prime} F}\left(U_{A^{\prime} \rightarrow B E}^{\mathcal{N}}\left(\rho_{A^{\prime}}\right)\right),
$$

with $U_{A^{\prime} \rightarrow B E}^{\mathcal{N}}$ an isometric extension of the channel $\mathcal{N}_{A^{\prime} \rightarrow B}$.

Proof. We prove this simply by manipulating the definition in (4). Consider a particular pure state $|\phi\rangle_{A A^{\prime}}$ and a squashing channel $\mathcal{S}_{E \rightarrow E^{\prime}}$. Let $\rho_{A^{\prime}}=\operatorname{Tr}_{A}\left\{\phi_{A A^{\prime}}\right\}$ and let $V_{E \rightarrow E^{\prime} F}$ be an isometric extension of the squashing channel $\mathcal{S}_{E \rightarrow E^{\prime}}$. Let $\omega_{A B E^{\prime} F} \equiv V_{E \rightarrow E^{\prime} F}\left(U_{A^{\prime} \rightarrow B E}^{\mathcal{N}}\left(\phi_{A A^{\prime}}\right)\right)$. Then

$$
\begin{aligned}
I\left(A ; B \mid E^{\prime}\right)_{\omega} & =H\left(B \mid E^{\prime}\right)_{\omega}-H\left(B \mid E^{\prime} A\right)_{\omega} \\
& =H\left(B \mid E^{\prime}\right)_{\omega}+H(B \mid F)_{\omega},
\end{aligned}
$$

where the first equality is an identity and the second follows from duality of conditional entropy (i.e., $H(K \mid L)=-H(K \mid M)$ for any pure tripartite state $\left.\psi_{K L M}\right)$. The statement of the lemma then holds because the above equality holds for any state $\phi_{A A^{\prime}}$ and any squashing channel $\mathcal{S}_{E \rightarrow E^{\prime}}$.

\subsection{Concavity in the input density operator}

Lemma 5 The squashed entanglement is concave in the input density operator $\rho_{A^{\prime}}$. That is, the following function (from Lemma 4) is concave as a function of $\rho_{A^{\prime}}$ :

$$
\frac{1}{2} \inf _{V_{E \rightarrow E^{\prime} F}}\left[H\left(B \mid E^{\prime}\right)_{\omega}+H(B \mid F)_{\omega}\right]
$$

where $\omega_{B E^{\prime} F} \equiv V_{E \rightarrow E^{\prime} F}\left(U_{A^{\prime} \rightarrow B E}^{\mathcal{N}}\left(\rho_{A^{\prime}}\right)\right)$.

Proof. Let $\rho_{A^{\prime}}=\sum_{x} p_{X}(x) \rho_{A^{\prime}}^{x}$ and let

$$
\omega_{X B E^{\prime} F} \equiv \sum_{x} p_{X}(x)|x\rangle\left\langle\left. x\right|_{X} \otimes V_{E \rightarrow E^{\prime} F}\left(U_{A^{\prime} \rightarrow B E}^{\mathcal{N}}\left(\rho_{A^{\prime}}^{x}\right)\right) .\right.
$$

Let $\omega_{B E^{\prime} F}^{x} \equiv V_{E \rightarrow E^{\prime} F}\left(U_{A^{\prime} \rightarrow B E}^{\mathcal{N}}\left(\rho_{A^{\prime}}^{x}\right)\right)$. Then the statement of the lemma is equivalent to

$$
\frac{1}{2} \inf _{V_{E \rightarrow E^{\prime} F}}\left[H\left(B \mid E^{\prime}\right)_{\omega}+H(B \mid F)_{\omega}\right] \geq \frac{1}{2} \sum_{x} p_{X}(x) \inf _{V_{E \rightarrow E^{\prime} F}^{x}}\left[H\left(B \mid E^{\prime}\right)_{\tau^{x}}+H(B \mid F)_{\tau^{x}}\right],
$$

where $\tau_{B E^{\prime} F}^{x} \equiv V_{E \rightarrow E^{\prime} F}^{x}\left(U_{A^{\prime} \rightarrow B E}^{\mathcal{N}}\left(\rho_{A^{\prime}}^{x}\right)\right)$. This follows from concavity of conditional entropy. That is, consider any state $\omega_{B E^{\prime} F}$ with fixed $\rho_{A^{\prime}}$ and fixed $U_{A^{\prime} \rightarrow B E}^{\mathcal{N}}$. Consider the following chain of inequalities:

$$
\begin{aligned}
H\left(B \mid E^{\prime}\right)_{\omega}+H(B \mid F)_{\omega} & \geq H\left(B \mid E^{\prime} X\right)_{\omega}+H(B \mid F X)_{\omega} \\
& =\sum_{x} p_{X}(x)\left[H\left(B \mid E^{\prime}\right)_{\omega^{x}}+H(B \mid F)_{\omega^{x}}\right] \\
& \geq \sum_{x} p_{X}(x) \inf _{V_{E \rightarrow E^{\prime} F}^{x}}\left[H\left(B \mid E^{\prime}\right)_{\tau^{x}}+H(B \mid F)_{\tau^{x}}\right]
\end{aligned}
$$


The first inequality follows from "conditioning cannot increase entropy" (i.e., $H(K \mid L) \geq H(K \mid L M)$ for any state on systems $K L M)$. The equality is just a rewriting of the entropies. The last inequality follows merely by taking an infimum over all squashing isometries corresponding to the individual states $U_{A^{\prime} \rightarrow B E}^{\mathcal{N}}\left(\rho_{A^{\prime}}^{x}\right)$. We can then conclude the statement of the lemma since the calculation is independent of which squashing isometry $V_{E \rightarrow E^{\prime} F}$ we begin with (i.e., it holds for the infimum).

With almost the same proof (excluding the last inequality above), we obtain the following:

Corollary 6 For a fixed squashing isometry $V_{E \rightarrow E^{\prime} F}$, the following function is concave in the input density operator $\rho_{A^{\prime}}$ :

$$
\frac{1}{2}\left[H\left(B \mid E^{\prime}\right)_{\omega}+H(B \mid F)_{\omega}\right]
$$

where $\omega_{B E^{\prime} F} \equiv V_{E \rightarrow E^{\prime} F}\left(U_{A^{\prime} \rightarrow B E}^{\mathcal{N}}\left(\rho_{A^{\prime}}\right)\right)$.

\subsection{Subadditivity inequality}

We now provide a statement and proof of the new subadditivity inequality:

Theorem 7 For any five-party pure state $\psi_{A B_{1} E_{1} B_{2} E_{2}}$, the following subadditivity inequality holds

$$
E_{\mathrm{sq}}\left(A ; B_{1} B_{2}\right)_{\psi} \leq E_{\mathrm{sq}}\left(A B_{2} E_{2} ; B_{1}\right)_{\psi}+E_{\mathrm{sq}}\left(A B_{1} E_{1} ; B_{2}\right)_{\psi}
$$

Proof. Let

$$
\begin{aligned}
\tau_{A B_{1} E_{1}^{\prime} B_{2} E_{2}} & \equiv \mathcal{S}_{E_{1} \rightarrow E_{1}^{\prime}}\left(\psi_{A B_{1} E_{1} B_{2} E_{2}}\right), \\
\sigma_{A B_{1} E_{1} B_{2} E_{2}^{\prime}} & \equiv \mathcal{S}_{E_{2} \rightarrow E_{2}^{\prime}}\left(\psi_{A B_{1} E_{1} B_{2} E_{2}}\right), \\
\omega_{A B_{1} E_{1}^{\prime} B_{2} E_{2}^{\prime}} & \equiv\left(\mathcal{S}_{E_{1} \rightarrow E_{1}^{\prime}} \otimes \mathcal{S}_{E_{2} \rightarrow E_{2}^{\prime}}\right)\left(\psi_{A B_{1} E_{1} B_{2} E_{2}}\right),
\end{aligned}
$$

where each $\mathcal{S}_{E_{i} \rightarrow E_{i}^{\prime}}$ is an arbitrary local squashing channel. Let $\left|\phi^{\omega}\right\rangle_{A B_{1} E_{1}^{\prime} B_{2} E_{2}^{\prime} R}$ be a purification of $\omega$ with purifying system $R$. The inequality in $(5)$ is a consequence of the following chain of inequalities:

$$
\begin{aligned}
2 E_{\mathrm{sq}}\left(A ; B_{1} B_{2}\right)_{\psi} & \leq I\left(A ; B_{1} B_{2} \mid E_{1}^{\prime} E_{2}^{\prime}\right)_{\omega} \\
& =H\left(B_{1} B_{2} \mid E_{1}^{\prime} E_{2}^{\prime}\right)_{\omega}-H\left(B_{1} B_{2} \mid E_{1}^{\prime} E_{2}^{\prime} A\right)_{\omega} \\
& =H\left(B_{1} B_{2} \mid E_{1}^{\prime} E_{2}^{\prime}\right)_{\phi}+H\left(B_{1} B_{2} \mid R\right)_{\phi} \\
& \leq H\left(B_{1} \mid E_{1}^{\prime}\right)_{\phi}+H\left(B_{2} \mid E_{2}^{\prime}\right)_{\phi}+H\left(B_{1} \mid R\right)_{\phi}+H\left(B_{2} \mid R\right)_{\phi} \\
& =H\left(B_{1} \mid E_{1}^{\prime}\right)_{\omega}-H\left(B_{1} \mid A B_{2} E_{1}^{\prime} E_{2}^{\prime}\right)_{\omega}+H\left(B_{2} \mid E_{2}^{\prime}\right)_{\omega}-H\left(B_{2} \mid A B_{1} E_{1}^{\prime} E_{2}^{\prime}\right)_{\omega} \\
& =I\left(A B_{2} E_{2}^{\prime} ; B_{1} \mid E_{1}^{\prime}\right)_{\omega}+I\left(A B_{1} E_{1}^{\prime} ; B_{2} \mid E_{2}^{\prime}\right)_{\omega} \\
& \leq I\left(A B_{2} E_{2} ; B_{1} \mid E_{1}^{\prime}\right)_{\tau}+I\left(A B_{1} E_{1} ; B_{2} \mid E_{2}^{\prime}\right)_{\sigma} .
\end{aligned}
$$

The first inequality follows from the definition in (3). The first equality is a rewriting of the conditional mutual information. The second equality exploits duality of conditional entropy: for any pure tripartite state on systems $K L M$, the equality $H(K \mid L)+H(K \mid M)=0$ holds. The second inequality results from several applications of strong subadditivity (SSA) of quantum entropy (SSA is the statement that $I(K ; L \mid M) \geq 0$ for an arbitrary state on systems $K L M)$ [LR73]. The third equality again exploits duality of conditional entropy and the last equality is just a rewriting in terms 
of conditional mutual informations. The final inequality is a result of a quantum data processing inequality for conditional mutual information (see the proof of Proposition 3 of [CW04]). Since the calculation above is independent of the choice of the maps $\mathcal{S}_{E_{i} \rightarrow E_{i}^{\prime}}$, the system $E_{1}$ purifies the state on $A B_{1} B_{2} E_{2}$, and the system $E_{2}$ purifies the state on $A B_{1} B_{2} E_{1}$, the subadditivity inequality in the statement of the theorem follows.

\subsection{Additivity}

As a simple corollary, we find that the squashed entanglement of a quantum channel is an additive, and thus well behaved, function of quantum channels.

Corollary 8 For any two quantum channels $\mathcal{N}$ and $\mathcal{M}$, the following additivity relation holds

$$
E_{\mathrm{sq}}(\mathcal{N} \otimes \mathcal{M})=E_{\mathrm{sq}}(\mathcal{N})+E_{\mathrm{sq}}(\mathcal{M})
$$

Proof. First, note that the following inequality holds

$$
E_{\mathrm{sq}}(\mathcal{N} \otimes \mathcal{M}) \geq E_{\mathrm{sq}}(\mathcal{N})+E_{\mathrm{sq}}(\mathcal{M})
$$

because the optimization of $E_{\mathrm{sq}}(\mathcal{N} \otimes \mathcal{M})$ includes tensor-product input choices as a special case and the squashed entanglement is additive for tensor-product states [CW04], so that $E_{\mathrm{sq}}(\mathcal{N} \otimes \mathcal{M})$ can only be larger than the sum of the individual terms.

The other inequality

$$
E_{\mathrm{sq}}(\mathcal{N} \otimes \mathcal{M}) \leq E_{\mathrm{sq}}(\mathcal{N})+E_{\mathrm{sq}}(\mathcal{M})
$$

follows from Theorem 7. Indeed, let $|\phi\rangle_{A A_{1} A_{2}}$ denote any pure-state input to the tensor product channel $\mathcal{N} \otimes \mathcal{M}$, so that the output state is $\left(\mathcal{N}_{A_{1} \rightarrow B_{1}} \otimes \mathcal{M}_{A_{2} \rightarrow B_{2}}\right)\left(|\phi\rangle\left\langle\left.\phi\right|_{A A_{1} A_{2}}\right)\right.$. Let $U_{A_{1} \rightarrow B_{1} E_{1}}^{\mathcal{N}}$ be an isometric extension of $\mathcal{N}_{A_{1} \rightarrow B_{1}}$ and let $V_{A_{2} \rightarrow B_{2} E_{2}}^{\mathcal{M}}$ be an isometric extension of $\mathcal{M}_{A_{2} \rightarrow B_{2}}$. Define the following states:

$$
\begin{aligned}
|\psi\rangle_{A B_{1} E_{1} A_{2}} & \equiv U_{A_{1} \rightarrow B_{1} E_{1}}^{\mathcal{N}}|\phi\rangle_{A A_{1} A_{2}} \\
|\chi\rangle_{A A_{1} B_{2} E_{2}} & \equiv V_{A_{2} \rightarrow B_{2} E_{2}}^{\mathcal{M}}|\phi\rangle_{A A_{1} A_{2}} \\
|\varphi\rangle_{A B_{1} E_{1} B_{2} E_{2}} & \equiv U_{A_{1} \rightarrow B_{1} E_{1}}^{\mathcal{N}} \otimes V_{A_{2} \rightarrow B_{2} E_{2}}^{\mathcal{M}}|\phi\rangle_{A A_{1} A_{2}}
\end{aligned}
$$

For any input state $|\phi\rangle_{A A_{1} A_{2}}$, the following holds

$$
\begin{aligned}
E_{\mathrm{sq}}\left(A ; B_{1} B_{2}\right)_{\varphi} & \leq E_{\mathrm{sq}}\left(A B_{2} E_{2} ; B_{1}\right)_{\varphi}+E_{\mathrm{sq}}\left(A B_{1} E_{1} ; B_{2}\right)_{\varphi} \\
& =E_{\mathrm{sq}}\left(A A_{2} ; B_{1}\right)_{\psi}+E_{\mathrm{sq}}\left(A A_{1} ; B_{2}\right)_{\chi} \\
& \leq E_{\mathrm{sq}}(\mathcal{N})+E_{\mathrm{sq}}(\mathcal{M}) .
\end{aligned}
$$

The first inequality is an application of Theorem 7. The equality follows because squashed entanglement is invariant under local isometries [CW04]. The final inequality follows because $|\phi\rangle_{A A_{1} A_{2}}$ is a particular pure-state input to the channel $\mathcal{N}_{A_{1} \rightarrow B_{1}}$ (with $A_{2}$ being the input and $A A_{1}$ being the purifying system), so that $E_{\mathrm{sq}}\left(A A_{2} ; B_{1}\right)_{\psi} \leq E_{\mathrm{sq}}(\mathcal{N})$, and a similar observation for the inequality $E_{\mathrm{sq}}\left(A A_{1} ; B_{2}\right)_{\chi} \leq E_{\mathrm{sq}}(\mathcal{M})$. Since the calculation is independent of which pure state $|\phi\rangle_{A A_{1} A_{2}}$ we begin with, the inequality in (6) follows. 


\section{Upper bound on capacities assisted by unlimited forward and backward communication}

The squashed entanglement of a quantum channel finds it main application in the theorems given in this section.

Theorem $9 E_{\mathrm{sq}}(\mathcal{N})$ is an upper bound on $Q_{2}(\mathcal{N})$, the quantum capacity of a channel $\mathcal{N}$ assisted by unlimited forward and backward classical communication:

$$
Q_{2}(\mathcal{N}) \leq E_{\mathrm{sq}}(\mathcal{N})
$$

Proof. First recall that the squashed entanglement is monotone under local operations and classical communication (LOCC), in the sense that $E_{\mathrm{sq}}(A ; B)_{\rho} \geq E_{\mathrm{sq}}(A ; B)_{\sigma}$ if Alice and Bob can obtain the state $\sigma_{A B}$ from $\rho_{A B}$ by LOCC [CW04. Furthermore, the squashed entanglement is normalized CW04, in the sense that $E_{\mathrm{sq}}(A ; B)_{\Phi}=\log d$ for a maximally entangled state defined as

$$
|\Phi\rangle_{A B} \equiv \frac{1}{\sqrt{d}} \sum_{i}|i\rangle_{A}|i\rangle_{B}
$$

where $\left\{|i\rangle_{A}\right\}$ and $\left\{|i\rangle_{B}\right\}$ are complete orthonormal bases for quantum systems $A$ and $B$, respectively. Finally, the squashed entanglement satisfies the following continuity inequality [AF04, Chr06]:

$$
\text { if }\left\|\rho_{A B}-\sigma_{A B}\right\|_{1} \leq \varepsilon, \text { then }\left|E_{\mathrm{sq}}(A ; B)_{\rho}-E_{\mathrm{sq}}(A ; B)_{\sigma}\right| \leq 16 \sqrt{\varepsilon} \log d+4 h_{2}(2 \sqrt{\varepsilon}),
$$

where $d=\min \{|A|,|B|\}$ and $h_{2}(x)$ is the binary entropy function with the property that $\lim _{x \rightarrow 0} h_{2}(x)=$ 0 . The most general $(n, R, \varepsilon)$ protocol in this setting begins with Alice preparing a state $\rho_{A A_{1} \cdots A_{n}}^{(1)}$ on $n+1$ systems. She then transmits the system $A_{1}$ through one use of the channel $\mathcal{N}$, and considering its isometric extension $U_{A_{1} \rightarrow B_{1} E_{1}}^{\mathcal{N}}$, we write the output state as $\sigma_{A B_{1} E_{1} A_{2} \cdots A_{n}}^{(1)}$. Let $R^{(1)}$ be a system that purifies this state. There is then a round of an arbitrary amount of LOCC between Alice and Bob, resulting in a state $\rho_{A B_{1} E_{1} A_{2} \cdots A_{n}}^{(2)}$. This procedure continues, with Alice transmitting system $A_{2}$ through the channel, leading to a state $\sigma_{A B_{1} E_{1} B_{2} E_{2} A_{3} \cdots A_{n}}^{(2)}$, etc. After the $n$th channel use, the state is $\sigma_{A B_{1} E_{1} B_{2} E_{2} \cdots B_{n} E_{n}}^{(n)}$ (note that the dimension of the system $A$ might change throughout the protocol). Let $R^{(n)}$ be a system that purifies this state. There is a final round of LOCC, producing a state $\omega_{A B E_{1} \cdots E_{n}}$, whose reduction $\omega_{A B}$ satisfies

$$
\| \omega_{A B}-|\Phi\rangle\left\langle\left.\Phi\right|_{A B} \|_{1} \leq \varepsilon,\right.
$$

where $|\Phi\rangle_{A B}$ is the maximally entangled state with Schmidt rank $2^{n R}$. We can now proceed by bounding the entanglement generation rate of any such protocol as follows:

$$
\begin{aligned}
n R & =E_{\mathrm{sq}}(A ; B)_{\Phi} \\
& \leq E_{\mathrm{sq}}(A ; B)_{\omega}+n f(\varepsilon) .
\end{aligned}
$$

The equality follows from the normalization of the squashed entanglement on maximally entangled states (as mentioned above). The inequality follows from continuity of squashed entanglement with 
an appropriate choice of $f(\varepsilon)$ so that $\lim _{\varepsilon \rightarrow 0} f(\varepsilon)=0$. Continuing,

$$
\begin{aligned}
E_{\mathrm{Sq}}(A ; B)_{\omega} \leq & E_{\mathrm{sq}}\left(A ; B_{1} \cdots B_{n}\right)_{\sigma^{(n)}} \\
\leq & E_{\mathrm{sq}}\left(A B_{1} E_{1} \cdots B_{n-1} E_{n-1} R^{(n)} ; B_{n}\right)_{\sigma^{(n)}} \\
& \quad+E_{\mathrm{sq}}\left(A B_{n} E_{n} ; B_{1} \cdots B_{n-1}\right)_{\sigma^{(n)}} \\
\leq & E_{\mathrm{sq}}(\mathcal{N})+E_{\mathrm{sq}}\left(A B_{n} E_{n} ; B_{1} \cdots B_{n-1}\right)_{\sigma^{(n)}} \\
= & E_{\mathrm{sq}}(\mathcal{N})+E_{\mathrm{sq}}\left(A A_{n} ; B_{1} \cdots B_{n-1}\right)_{\rho^{(n)}} \\
\leq & n E_{\mathrm{sq}}(\mathcal{N}) .
\end{aligned}
$$

The first inequality follows from monotonicity of the squashed entanglement under LOCC. The second inequality is an application of the subadditivity inequality in Theorem 7. The third inequality follows because $E_{\mathrm{sq}}\left(A B_{1} E_{1} \cdots B_{n-1} E_{n-1} R^{(n)} ; B_{n}\right)_{\sigma^{(n)}} \leq E_{\mathrm{sq}}(\mathcal{N})$ (there is a particular input to the $n$th channel, while the systems $A B_{1} E_{1} \cdots B_{n-1} E_{n-1} R^{(n)}$ purify the system being input to the channel). The sole equality follows because the squashed entanglement is invariant under local isometries (the isometry here being the isometric extension of the channel). The last inequality follows by induction, i.e., repeating this procedure by using monotonicity under LOCC and subadditivity, "peeling off" one term at a time. Putting everything together, we arrive at

$$
n R \leq n E_{\mathrm{sq}}(\mathcal{N})+n f(\varepsilon),
$$

which we can divide by $n$ and take the limit as $\varepsilon \rightarrow 0$ to recover the result that $Q_{2}(\mathcal{N}) \leq E_{\mathrm{sq}}(\mathcal{N})$.

Remark 10 Observe that, in spite of the fact that the squashed entanglement of a quantum channel is difficult to compute exactly, it is useful in obtaining upper bounds on the assisted capacities $Q_{2}(\mathcal{N})$ and $P_{2}(\mathcal{N})$ (see Theorem 12 below for $P_{2}(\mathcal{N})$ ) because any squashing channel leads to an upper bound.

Remark 11 Just as the squashed entanglement of a channel $\mathcal{N}$ serves as an upper bound on $Q_{2}(\mathcal{N})$, we can in fact find other single-letter upper bounds on $Q_{2}(\mathcal{N})$ from any function on quantum states that satisfies LOCC monotonicity, asymptotic continuity, normalization (it equals $\log d$ for a maximally entangled state of dimension d), invariance under local unitaries, and the subadditivity inequality in Theorem 7 . This follows because these were the only properties of $E_{\mathrm{sq}}(\mathcal{N})$ that we used to prove the above theorem.

A variation of this setting is one in which there is a forward quantum channel $\mathcal{N}$ connecting Alice to Bob and a backward quantum channel $\mathcal{M}$ connecting Bob to Alice. The most general protocol for communicating quantum data (or equivalently in this setting, generating entanglement) has Alice and Bob each prepare a state on $n$ systems, Alice sends one system through the forward channel, they conduct a round of LOCC, Bob sends one of his systems through the backward channel, they conduct a round of LOCC, etc. By essentially the same proof technique as above, it follows that $E_{\mathrm{sq}}(\mathcal{N})+E_{\mathrm{sq}}(\mathcal{M})$ is an upper bound on the total rate of quantum communication they can generate with these channels.

Theorem 12 The squashed entanglement $E_{\mathrm{sq}}(\mathcal{N})$ serves as an upper bound on the secret-key agreement capacity $P_{2}(\mathcal{N})$ of a quantum channel $\mathcal{N}$ :

$$
P_{2}(\mathcal{N}) \leq E_{\mathrm{sq}}(\mathcal{N})
$$


Proof. Christandl et al. showed in prior work that the squashed entanglement is a secrecy monotone, in the sense that it does not increase under local operations and public classical (LOPC) communication [Chr06, $\left.\mathrm{CEH}^{+} 07\right]$. The method for doing so was to exploit the fact that LOPC distillation of secret key is equivalent to LOCC distillation of private states [HHHO05, HHHO09]. Combining this with the fact that squashed entanglement is normalized, in the sense that it is never smaller than $k$ for a $k$-bit private state (see Proposition 4.19 of [Chr06]), and a proof essentially identical to the proof of Theorem 9 , we recover that $P_{2}(\mathcal{N}) \leq E_{\mathrm{sq}}(\mathcal{N})$.

By similar arguments as above, the secret-key agreement capacity is upper bounded by $E_{\mathrm{sq}}(\mathcal{N})+$ $E_{\text {sq }}(\mathcal{M})$ in the setting where there is a forward quantum channel $\mathcal{N}$ and a backward quantum channel $\mathcal{M}$.

Remark 13 Just as the squashed entanglement of a channel $\mathcal{N}$ serves as an upper bound on $P_{2}(\mathcal{N})$, we can find other single-letter upper bounds on it from any function on quantum states that satisfies LOCC monotonicity, asymptotic continuity, normalization (it is never smaller than $k$ for a $k$-bit private state), invariance under local unitaries, and the subadditivity inequality in Theorem 7 . Again, this follows because these were the only properties that we used to prove the above theorem.

Remark 14 The squashed entanglement of a quantum channel is never larger than its entanglement cost [BBCW13]. This is an immediate consequence of Corollary 6 of [CW04].

\section{Application to Pauli channels}

In this section, we apply Theorems 9 and 12 to the case of a Pauli channel. That is, we establish an upper bound on $Q_{2}(\mathcal{P})$ and $P_{2}(\mathcal{P})$ where $\mathcal{P}$ is a Pauli channel, defined as

$$
\mathcal{P}(\rho)=p_{0} \rho+p_{1} X \rho X+p_{2} Y \rho Y+p_{3} Z \rho Z .
$$

In the above, the probabilities $p_{i}$ are non-negative, $\sum_{i} p_{i}=1$, and

$$
I=\left[\begin{array}{ll}
1 & 0 \\
0 & 1
\end{array}\right], \quad X=\left[\begin{array}{ll}
0 & 1 \\
1 & 0
\end{array}\right], \quad Y=\left[\begin{array}{cc}
0 & -i \\
i & 0
\end{array}\right], \quad Z=\left[\begin{array}{cc}
1 & 0 \\
0 & -1
\end{array}\right]
$$

are the Pauli operators. We also denote the Pauli operators by $\sigma^{0}, \ldots, \sigma^{3}$, respectively. Note that the Pauli channel is equivalent to

$$
\mathcal{P}(\rho)=p_{0} \rho+p_{1} X \rho X+p_{2} X Z \rho Z X+p_{3} Z \rho Z,
$$

due to the fact that $Y=i X Z$ and $X Z=-Z X$.

Theorem 15 The squashed entanglement leads to the following upper bound on $Q_{2}(\mathcal{P})$ and $P_{2}(\mathcal{P})$ :

$$
Q_{2}(\mathcal{P}), P_{2}(\mathcal{P}) \leq \min _{\varphi_{1}, \varphi_{2}, \varphi_{3}} \frac{1}{2}\left[H(\lambda)+H\left(\lambda^{\prime}\right)\right]-1
$$


where $\mathcal{P}$ is a Pauli channel and $H(\lambda)$ is the Shannon entropy of the distribution $\lambda=\left\{\lambda_{0}, \lambda_{1}, \lambda_{2}, \lambda_{3}\right\}$, with

$$
\begin{aligned}
& \lambda_{0}=\frac{1}{4}\left|\sqrt{p_{0}}+e^{i \varphi_{3}} \sqrt{p_{3}}+e^{i \varphi_{1}} \sqrt{p_{1}}-e^{i \varphi_{2}} \sqrt{p_{2}}\right|^{2}, \\
& \lambda_{1}=\frac{1}{4}\left|\sqrt{p_{0}}+e^{i \varphi_{3}} \sqrt{p_{3}}-e^{i \varphi_{1}} \sqrt{p_{1}}+e^{i \varphi_{2}} \sqrt{p_{2}}\right|^{2}, \\
& \lambda_{2}=\frac{1}{4}\left|\sqrt{p_{0}}-e^{i \varphi_{3}} \sqrt{p_{3}}+e^{i \varphi_{1}} \sqrt{p_{1}}+e^{i \varphi_{2}} \sqrt{p_{2}}\right|^{2}, \\
& \lambda_{3}=\frac{1}{4}\left|-\sqrt{p_{0}}+e^{i \varphi_{3}} \sqrt{p_{3}}+e^{i \varphi_{1}} \sqrt{p_{1}}+e^{i \varphi_{2}} \sqrt{p_{2}}\right|^{2},
\end{aligned}
$$

and $\lambda^{\prime}$ is the same as $\lambda$ except with the substitution $\varphi_{2} \rightarrow \varphi_{2}+\pi$.

Proof. Let

$$
\left|\Phi^{ \pm}\right\rangle=\frac{1}{\sqrt{2}}(|00\rangle \pm|11\rangle), \quad\left|\Psi^{ \pm}\right\rangle=\frac{1}{\sqrt{2}}(|01\rangle \pm|10\rangle)
$$

denote the four Bell states.

An isometric extension of the Pauli channel is as follows: the environment prepares a Bell state $\left|\Phi^{+}\right\rangle_{E F}$ and interacts qubit $E$ with the input state (in system $A^{\prime}$ ) according to a controlled Pauli gate. That is, this isometric extension $W_{A^{\prime} \rightarrow B E F}$ of $\mathcal{P}_{A^{\prime} \rightarrow B}$ acting on an input $|\psi\rangle_{A^{\prime}}$ is as follows:

$$
\begin{aligned}
W_{A^{\prime} \rightarrow B E F}|\psi\rangle_{A^{\prime}}=\sqrt{p_{0}}|\psi\rangle_{B}\left|\Phi^{+}\right\rangle_{E F}+\sqrt{p_{1}} e^{i \varphi_{1}} X|\psi\rangle_{B}\left|\Psi^{+}\right\rangle_{E F} \\
+\sqrt{p_{2}} e^{i \varphi_{2}} X Z|\psi\rangle_{B}\left|\Psi^{-}\right\rangle_{E F}+e^{i \varphi_{3}} Z|\psi\rangle_{B}\left|\Phi^{-}\right\rangle_{E F},
\end{aligned}
$$

where $\varphi_{1,2,3}$ are free parameters that we can choose later (we already take $\varphi_{0}=0$ because invariance of quantum states under a global phase eliminates one of these degrees of freedom). Note that tracing out systems $E$ and $F$ from (14) gives (7) with $|\psi\rangle$ as input (meaning that (14) is a legitimate isometric extension). Let the squashing channel consist of tracing over system $F$.

We now argue that if the channel's isometry and the corresponding squashing channel are fixed to be as above, then the optimal input state on systems $A A^{\prime}$ to maximize the conditional mutual information $I(A ; B \mid E)$ is the maximally entangled state $\left|\Phi^{+}\right\rangle_{A A^{\prime}}$. Indeed, consider that the Pauli channel is covariant, so that for all Pauli operators $U$ there exists a unitary $V$ such that

$$
\mathcal{P}(U(\psi))=V(\mathcal{P}(\psi)) .
$$

Let $\mathcal{P}^{1}(\cdot)=\operatorname{Tr}_{F}\left\{W(\cdot) W^{\dagger}\right\}$ and $\mathcal{P}^{2}(\cdot)=\operatorname{Tr}_{E}\left\{W(\cdot) W^{\dagger}\right\}$. Both of these channels are covariant, in the sense that for all Pauli operators $U$, there exist unitaries $V_{B}^{1}$ and $V_{E}^{1}$ such that

$$
\mathcal{P}^{1}(U(\psi))=\left(V_{B}^{1} \otimes V_{E}^{1}\right)(\mathcal{P}(\psi))\left(V_{B}^{1} \otimes V_{E}^{1}\right)^{\dagger} .
$$

Similarly, for all Pauli operators $U$, there exist unitaries $V_{B}^{2}$ and $V_{F}^{2}$ such that

$$
\mathcal{P}^{2}(U(\psi))=\left(V_{B}^{2} \otimes V_{F}^{2}\right)(\mathcal{P}(\psi))\left(V_{B}^{2} \otimes V_{F}^{2}\right)^{\dagger} .
$$

Equivalently, by inspecting (14), we see that

$$
\begin{aligned}
& W_{A^{\prime} \rightarrow B E F} X_{A^{\prime}}|\psi\rangle_{A^{\prime}}=\left(X_{B} \otimes X_{E} \otimes X_{F}\right) W_{A^{\prime} \rightarrow B E F}|\psi\rangle_{A^{\prime}}, \\
& W_{A^{\prime} \rightarrow B E F} Z_{A^{\prime}}|\psi\rangle_{A^{\prime}}=\left(Z_{B} \otimes Z_{E} \otimes Z_{F}\right) W_{A^{\prime} \rightarrow B E F}|\psi\rangle_{A^{\prime}},
\end{aligned}
$$


and from this observation (extended by linearity), the covariance stated above follows. This means that

$$
H(B \mid E)_{\omega}+H(B \mid F)_{\omega}=H(B \mid E)_{\tau}+H(B \mid F)_{\tau},
$$

where $\omega$ is the state resulting from preparing a state $\rho_{A^{\prime}}$ at the input $A^{\prime}$ and $\tau$ is the state resulting from preparing $U \rho_{A^{\prime}} U^{\dagger}$, with $U$ any Pauli operator. We can then apply Corollary 6 (concavity of $H(B \mid E)+H(B \mid F)$ in the input density operator) to conclude that the maximizing input density operator is the maximally mixed state, since $I / 2=\frac{1}{4} \sum_{i=0}^{3} \sigma^{i} \rho \sigma^{i}$ for any input state $\rho$. Since the maximally mixed state on $A^{\prime}$ is purified by the maximally entangled state $\left|\Phi^{+}\right\rangle_{A A^{\prime}}$, we conclude that the maximally entangled state maximizes $I(A ; B \mid E)$ whenever the channel isometry and squashing channel are fixed to be of the form in (14) (if the channel isometry is not of this form, then we can take the squashing channel to consist of a preliminary isometric rotation to make it have the above form, followed by a tracing out of system $F$ ).

We now evaluate the squashed entanglement upper bound using the above squashing channel. For this purpose, we recall Lemma 4, which expresses the squashed entanglement as

$$
\frac{1}{2}[H(B \mid E)+H(B \mid F)]
$$

So we need to compute the eigenvalues of various reduced density matrices in order to evaluate the above entropies. To derive the reduced density operator $\rho_{B E}$, we factorize the systems $A$ and $F$ of the state $W_{A^{\prime} \rightarrow B E F}\left|\Phi^{+}\right\rangle_{A A^{\prime}}$ as follows:

$$
\begin{aligned}
\sqrt{p_{0}}\left|\Phi_{+}\right\rangle_{A B}\left|\Phi_{+}\right\rangle_{E F}+e^{i \varphi_{3}} \sqrt{p_{3}}\left|\Phi_{-}\right\rangle_{A B}\left|\Phi_{-}\right\rangle_{E F}+e^{i \varphi_{1}} \sqrt{p_{1}}\left|\Psi_{+}\right\rangle_{A B}\left|\Psi_{+}\right\rangle_{E F}+e^{i \varphi_{2}} \sqrt{p_{2}}\left|\Psi_{-}\right\rangle_{A B}\left|\Psi_{-}\right\rangle_{E F} \\
=\frac{1}{\sqrt{2}}|0\rangle_{A}\left(\sqrt{p_{0}}|0\rangle_{B}\left|\Phi_{+}\right\rangle_{E F}+e^{i \varphi_{3}} \sqrt{p_{3}}|0\rangle_{B}\left|\Phi_{-}\right\rangle_{E F}+e^{i \varphi_{1}} \sqrt{p_{1}}|1\rangle_{B}\left|\Psi_{+}\right\rangle_{E F}+e^{i \varphi_{2}} \sqrt{p_{2}}|1\rangle_{B}\left|\Psi_{-}\right\rangle_{E F}\right) \\
\quad+\frac{1}{\sqrt{2}}|1\rangle_{A}\left(\sqrt{p_{0}}|1\rangle_{B}\left|\Phi_{+}\right\rangle_{E F}-e^{i \varphi_{3}} \sqrt{p_{3}}|1\rangle_{B}\left|\Phi_{-}\right\rangle_{E F}+e^{i \varphi_{1}} \sqrt{p_{1}}|0\rangle_{B}\left|\Psi_{+}\right\rangle_{E F}-e^{i \varphi_{2}} \sqrt{p_{2}}|0\rangle_{B}\left|\Psi_{-}\right\rangle_{E F}\right) \\
=\frac{1}{2}|0\rangle_{A}|0\rangle_{F}\left(\sqrt{p_{0}}|00\rangle+e^{i \varphi_{3}} \sqrt{p_{3}}|00\rangle+e^{i \varphi_{1}} \sqrt{p_{1}}|11\rangle-e^{i \varphi_{2}} \sqrt{p_{2}}|11\rangle\right)_{B E} \\
\quad+\frac{1}{2}|0\rangle_{A}|1\rangle_{F}\left(\sqrt{p_{0}}|01\rangle-e^{i \varphi_{3}} \sqrt{p_{3}}|01\rangle+e^{i \varphi_{1}} \sqrt{p_{1}}|10\rangle+e^{i \varphi_{2}} \sqrt{p_{2}}|10\rangle\right)_{B E} \\
\quad+\frac{1}{2}|1\rangle_{A}|0\rangle_{F}\left(\sqrt{p_{0}}|10\rangle-e^{i \varphi_{3}} \sqrt{p_{3}}|10\rangle+e^{i \varphi_{1}} \sqrt{p_{1}}|01\rangle+e^{i \varphi_{2}} \sqrt{p_{2}}|01\rangle\right)_{B E} \\
\quad+\frac{1}{2}|1\rangle_{A}|1\rangle_{F}\left(\sqrt{p_{0}}|11\rangle+e^{i \varphi_{3}} \sqrt{p_{3}}|11\rangle+e^{i \varphi_{1}} \sqrt{p_{1}}|00\rangle-e^{i \varphi_{2}} \sqrt{p_{2}}|00\rangle\right)_{B E} .
\end{aligned}
$$

Tracing out $A$ and $F$, we have

$$
\begin{aligned}
\rho_{B E}=\frac{1}{4}\left[\left\{\left(\sqrt{p_{0}}+\right.\right.\right. & \left.\left.e^{i \varphi_{3}} \sqrt{p_{3}}\right)|00\rangle+\left(e^{i \varphi_{1}} \sqrt{p_{1}}-e^{i \varphi_{2}} \sqrt{p_{2}}\right)|11\rangle\right\}\{h . c .\} \\
& +\left\{\left(\sqrt{p_{0}}+e^{i \varphi_{3}} \sqrt{p_{3}}\right)|11\rangle+\left(e^{i \varphi_{1}} \sqrt{p_{1}}-e^{i \varphi_{2}} \sqrt{p_{2}}\right)|00\rangle\right\}\{h . c .\} \\
& +\left\{\left(\sqrt{p_{0}}-e^{i \varphi_{3}} \sqrt{p_{3}}\right)|01\rangle+\left(e^{i \varphi_{1}} \sqrt{p_{1}}+e^{i \varphi_{2}} \sqrt{p_{2}}\right)|10\rangle\right\}\{h . c .\} \\
& \left.+\left\{\left(\sqrt{p_{0}}-e^{i \varphi_{3}} \sqrt{p_{3}}\right)|10\rangle+\left(e^{i \varphi_{1}} \sqrt{p_{1}}+e^{i \varphi_{2}} \sqrt{p_{2}}\right)|01\rangle\right\}\{\text { h.c. }\}\right],
\end{aligned}
$$

which consists of two block diagonalized submatrices in the subspaces spanned by $\{|00\rangle,|11\rangle\}$ and $\{|01\rangle,|10\rangle\}$, respectively:

$$
=\frac{1}{4}\left[\begin{array}{cc}
|a|^{2}+|b|^{2} & a b^{*}+a^{*} b \\
a b^{*}+a^{*} b & |a|^{2}+|b|^{2}
\end{array}\right] \oplus \frac{1}{4}\left[\begin{array}{cc}
|c|^{2}+|d|^{2} & c d^{*}+c^{*} d \\
c d^{*}+c^{*} d & |c|^{2}+|d|^{2}
\end{array}\right],
$$


where

$$
\begin{aligned}
& a=\sqrt{p_{0}}+e^{i \varphi_{3}} \sqrt{p_{3}}, \\
& b=e^{i \varphi_{1}} \sqrt{p_{1}}-e^{i \varphi_{2}} \sqrt{p_{2}}, \\
& c=\sqrt{p_{0}}-e^{i \varphi_{3}} \sqrt{p_{3}}, \\
& d=e^{i \varphi_{1}} \sqrt{p_{1}}+e^{i \varphi_{2}} \sqrt{p_{2}} .
\end{aligned}
$$

This is diagonalized by the following unitary transformation:

$$
U=\frac{1}{\sqrt{2}}\left[\begin{array}{cc}
1 & 1 \\
1 & -1
\end{array}\right] \oplus \frac{1}{\sqrt{2}}\left[\begin{array}{cc}
1 & 1 \\
1 & -1
\end{array}\right]
$$

so that

$$
U \rho_{B E} U^{\dagger}=\frac{1}{4}\left[\begin{array}{cc}
|a+b|^{2} & 0 \\
0 & |a-b|^{2}
\end{array}\right] \oplus \frac{1}{4}\left[\begin{array}{cc}
|c+d|^{2} & 0 \\
0 & |c-d|^{2}
\end{array}\right] .
$$

Thus we find the following four eigenvalues for $\rho_{B E}$ :

$$
\begin{aligned}
& \lambda_{0}=\frac{1}{4}\left|\sqrt{p_{0}}+e^{i \varphi_{3}} \sqrt{p_{3}}+e^{i \varphi_{1}} \sqrt{p_{1}}-e^{i \varphi_{2}} \sqrt{p_{2}}\right|^{2}, \\
& \lambda_{1}=\frac{1}{4}\left|\sqrt{p_{0}}+e^{i \varphi_{3}} \sqrt{p_{3}}-e^{i \varphi_{1}} \sqrt{p_{1}}+e^{i \varphi_{2}} \sqrt{p_{2}}\right|^{2}, \\
& \lambda_{2}=\frac{1}{4}\left|\sqrt{p_{0}}-e^{i \varphi_{3}} \sqrt{p_{3}}+e^{i \varphi_{1}} \sqrt{p_{1}}+e^{i \varphi_{2}} \sqrt{p_{2}}\right|^{2}, \\
& \lambda_{3}=\frac{1}{4}\left|-\sqrt{p_{0}}+e^{i \varphi_{3}} \sqrt{p_{3}}+e^{i \varphi_{1}} \sqrt{p_{1}}+e^{i \varphi_{2}} \sqrt{p_{2}}\right|^{2},
\end{aligned}
$$

from which we can calculate the von Neumann entropy as

$$
H(B E)_{\rho}=H(\lambda)
$$

where $H(\lambda)$ is the Shannon entropy of the distribution $\lambda=\left\{\lambda_{0}, \lambda_{1}, \lambda_{2}, \lambda_{3}\right\}$.

Tracing over the $B$ system results in the maximally mixed state on the $E$ system, so that

$$
H(E)=1 .
$$

Similarly, we find that the reduced state on system $F$ is maximally mixed, so that $H(F)=1$. Now, if we instead trace over systems $A$ and $E$, the calculation of the eigenvalues of the reduced density matrix on systems $B$ and $F$ is similar to that detailed above. However, observe that all of the Bell states are invariant under a swap, with the exception of $\left|\Psi^{-}\right\rangle$. So, starting from (15), we realize that the eigenvalues are the same, except we have the substitution $\varphi_{2} \rightarrow \varphi_{2}+\pi$ due to the previous observation. We then recover the statement of the theorem.

As a lower bound on both $Q_{2}(\mathcal{P})$ and $P_{2}(\mathcal{P})$, both the direct and the reverse coherent information [GPLS09] for a Pauli channel with the Bell state input are given by

$$
I_{R}\left(\mathcal{N}_{P}, \Phi^{+}\right)=H(A)_{\rho}-H(A B)_{\rho}=\max \{0,1-H(\mathbf{p})\}
$$

where $\mathbf{p}=\left\{p_{0}, p_{1}, p_{2}, p_{3}\right\}$ 


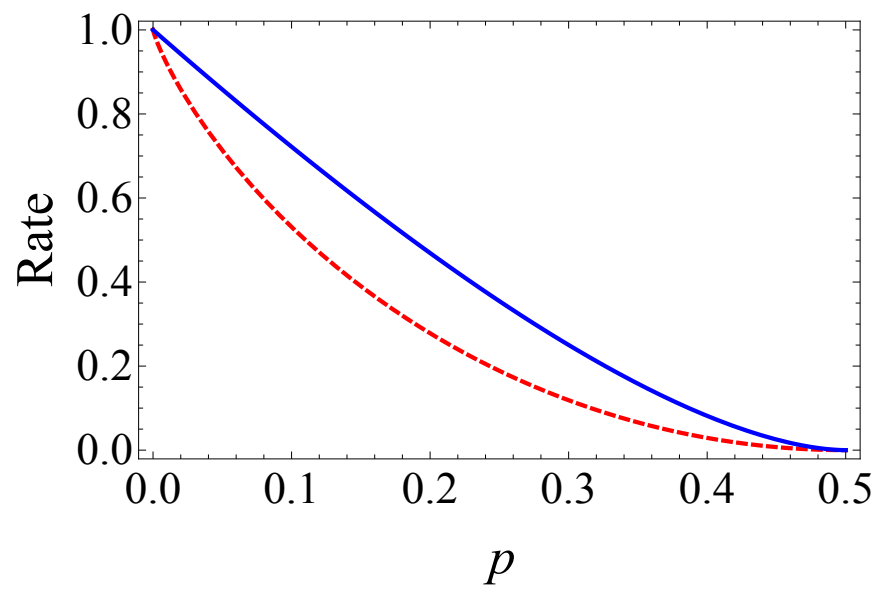

Figure 1: Upper and lower bounds on $Q_{2}$ and $P_{2}$ for a qubit dephasing channel. The dashed curve is both the direct and reverse coherent information, while the solid curve is the squashed entanglement upper bound. Note that this latter curve is equal to the entanglement cost from BBCW13] for this particular channel.

\subsection{Dephasing channel}

The dephasing channel $\mathcal{D}$ is obtained from a Pauli channel by setting $p_{1}=p_{2}=0$. The eigenvalues of $\rho_{B E}$ in this case reduce to

$$
\begin{aligned}
& \lambda_{0,1}=\frac{1}{4}\left(1+2 \sqrt{p(1-p)} \cos \varphi_{3}\right), \\
& \lambda_{2,3}=\frac{1}{4}\left(1-2 \sqrt{p(1-p)} \cos \varphi_{3}\right),
\end{aligned}
$$

where we set $p=p_{0}$. Clearly, $H(\lambda)=H\left(\lambda^{\prime}\right)$ and $H(\lambda)$ is minimized by setting $\varphi_{3}=0$. So our upper bound becomes

$$
Q_{2}(\mathcal{D}), P_{2}(\mathcal{D}) \leq h_{2}\left(\frac{1+2 \sqrt{p(1-p)}}{2}\right),
$$

where $h_{2}(\cdot)$ is the binary entropy function. Note that the above bound is equal to the entanglement cost of the dephasing channel (compare with (83) of [BBCW13]). Both the direct and the reverse coherent information for a dephasing channel are given by $I_{R}=1-h_{2}(p)$.

\subsection{Depolarizing channel}

The depolarizing channel is also a special case of a Pauli channel:

$$
\begin{aligned}
\mathcal{N}_{\text {dep }}(\rho) & =(1-p) \rho+p \frac{I}{2} \\
& =\left(1-\frac{3 p}{4}\right) \rho+\frac{p}{4}(X \rho X+Y \rho Y+Z \rho Z) .
\end{aligned}
$$




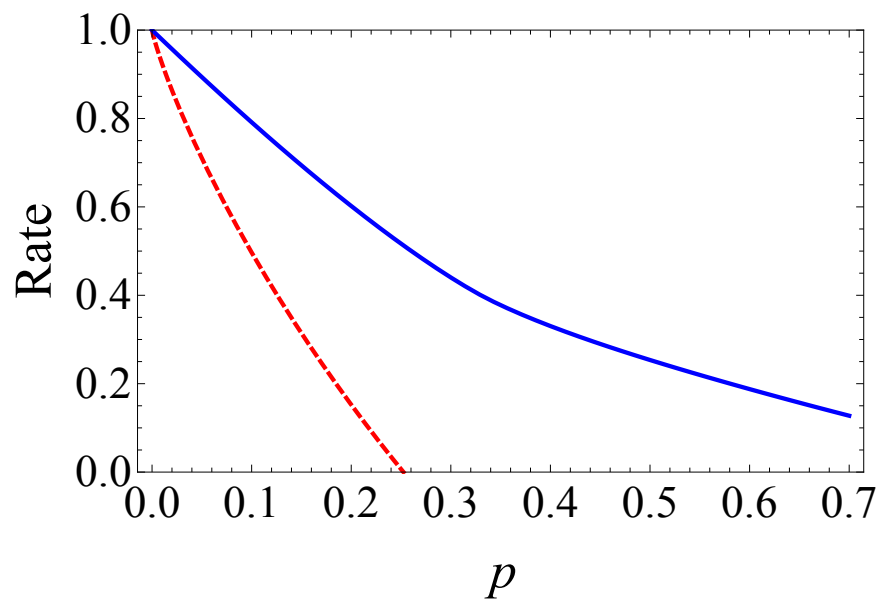

Figure 2: Upper and lower bounds on $Q_{2}$ and $P_{2}$ for a qubit depolarizing channel. The dashed curve is both the direct and reverse coherent information, while the solid curve is the squashed entanglement upper bound.

Numerical work indicates that the minimizing choice for the phases $\varphi_{1}, \varphi_{2}$, and $\varphi_{3}$ from Theorem 15 is simply $\varphi_{1}=\varphi_{2}=\varphi_{3}=0$. Figure 2 plots the squashed entanglement upper bound and both the direct and the reverse coherent information for this channel. This figure makes it clear that our squashed entanglement upper bound is not particularly tight in this case because the qubit depolarizing channel becomes entanglement-breaking whenever $p \geq 2 / 3$ and thus $Q_{2}\left(\mathcal{N}_{\text {dep }}\right)=0$ whenever $p \geq 2 / 3$ [Rai01].

\section{Application to bosonic channels}

\subsection{Pure-loss channel}

As a final contribution, we apply our bounds to the practically relevant pure-loss bosonic channel, which is modeled by the following Heisenberg picture evolution:

$$
\hat{b}=\sqrt{\eta} \hat{a}+\sqrt{1-\eta} \hat{e},
$$

where $\hat{a}, \hat{b}$, and $\hat{e}$ are the electromagnetic field mode operators corresponding to the sender's input, the receiver's output, and the environmental input. For the pure-loss bosonic channel, the environment injects the vacuum state. The parameter $\eta \in[0,1]$ characterizes the transmissivity of the channel, i.e., the fraction of input photons that make it to the output on average. Let $\mathcal{N}_{\eta}$ denote the channel to the receiver.

For a classical-communication-assisted quantum communication protocol or secret-key agreement protocol over such a channel, we assume that it begins and ends with finite-dimensional states, but the processing between the first and final step can be with infinite-dimensional systems.2 ${ }^{2}$ Furthermore, we impose a mean photon number constraint at the input of each channel, i.e., for each

\footnotetext{
${ }^{2}$ That is, their objective is to generate a maximally entangled state $|\Phi\rangle_{A B}$ or a finite number of secret key bits, and they do so by Alice encoding a finite-dimensional quantum state into an infinite-dimensional system and the final step of the protocol has them truncate their systems to be of finite dimension. In this way, the continuity inequality in
} 
channel input, we have the constraint that $\left\langle\hat{a}^{\dagger} \hat{a}\right\rangle \leq N_{S}$ for some $N_{S}$ such that $0 \leq N_{S}<\infty$. Thus, $E_{\mathrm{sq}}\left(\mathcal{N}_{\eta}\right)$ with the additional photon number constraint on the channel input is an upper bound on both $Q_{2}\left(\mathcal{N}_{\eta}\right)$ and $P_{2}\left(\mathcal{N}_{\eta}\right)$. By taking the squashing channel for the environment to be another pure-loss bosonic channel of transmissivity $\eta_{1} \in[0,1]$, noting that the resulting conditional mutual information can be written as a sum of two conditional entropies as in Lemma 4 , and applying the extremality of Gaussian states with respect to conditional entropies [EW07, WGC06], we find the following upper bounds on $E_{\mathrm{sq}}\left(\mathcal{N}_{\eta}\right)$ for all $\eta_{1} \in[0,1]$ (see Appendix A for a detailed proof):

$$
\frac{1}{2}\left[g\left(\left(1-\eta_{1}+\eta \eta_{1}\right) N_{S}\right)+g\left(\left(\eta_{1}+\eta\left(1-\eta_{1}\right)\right) N_{S}\right)-g\left(\eta_{1}(1-\eta) N_{S}\right)-g\left(\left(1-\eta_{1}\right)(1-\eta) N_{S}\right)\right],
$$

where $g(x) \equiv(x+1) \log _{2}(x+1)-x \log _{2} x$ is the entropy of a bosonic, circularly-symmetric thermal state with mean photon number $x$. The function in 26 is symmetric and convex in $\eta_{1}$ (see Appendix A.1), so that its minimum occurs at $\eta_{1}=1 / 2$, leading to the following simpler upper bound:

$$
g\left((1+\eta) N_{S} / 2\right)-g\left((1-\eta) N_{S} / 2\right)
$$

By taking the limit of this upper bound as $N_{S} \rightarrow \infty$, we recover the following photon-number independent upper bound on the capacities $Q_{2}\left(\mathcal{N}_{\eta}\right)$ and $P_{2}\left(\mathcal{N}_{\eta}\right)$ :

$$
\log _{2}\left(\frac{1+\eta}{1-\eta}\right)
$$

For values of $\eta \ll 1$ (which we expect in practical scenarios with high loss), this upper bound is close to the following lower bound on $Q_{2}\left(\mathcal{N}_{\eta}\right)$ and $P_{2}\left(\mathcal{N}_{\eta}\right)$ established in [GPLS09, PGBL09]:

$$
\log _{2}\left(\frac{1}{1-\eta}\right)
$$

Thus, for such small $\eta$, our upper bound demonstrates that the protocols from [GPLS09, PGBL09] achieving the lower bound in (28) are nearly optimal. Figure 3 plots these bounds.

Remark 16 In [HW01], Holevo and Werner proved by a different approach that $\log _{2}((1+\eta) /(1-\eta))$ serves as an upper bound on the unassisted quantum capacity of a pure-loss bosonic channel with transmissivity $\eta \in[0,1]$. However, it is not clear to us that their method generalizes to yield an upper bound on the quantum capacity assisted by unlimited forward and backward classical communication. Furthermore, in light of later results [WPGG07] which established $\log _{2}(\eta /(1-\eta))$ as an upper bound on the unassisted quantum capacity, it is clear that the Holevo-Werner bound is not tight.

\subsection{Phase-insensitive Gaussian channels}

In this section, we find bounds on $Q_{2}(\mathcal{N})$ and $P_{2}(\mathcal{N})$ whenever $\mathcal{N}$ is a phase-insensitive Gaussian channel $\left.\mathrm{WPGP}^{+} 12, \mathrm{GPNBL}^{+} 12\right]$, meaning that it adds an equal amount of noise to each quadrature of the electromagnetic field. Examples of these channels include the pure-loss channel, the thermal channel, the additive noise channel, and the phase-insensitive amplifier channel.

the proof of Theorem 9 safely applies and all of the other steps in between involve only the quantum data processing inequality, which has been proven to hold in the general infinite-dimensional setting [Uhl77]. 


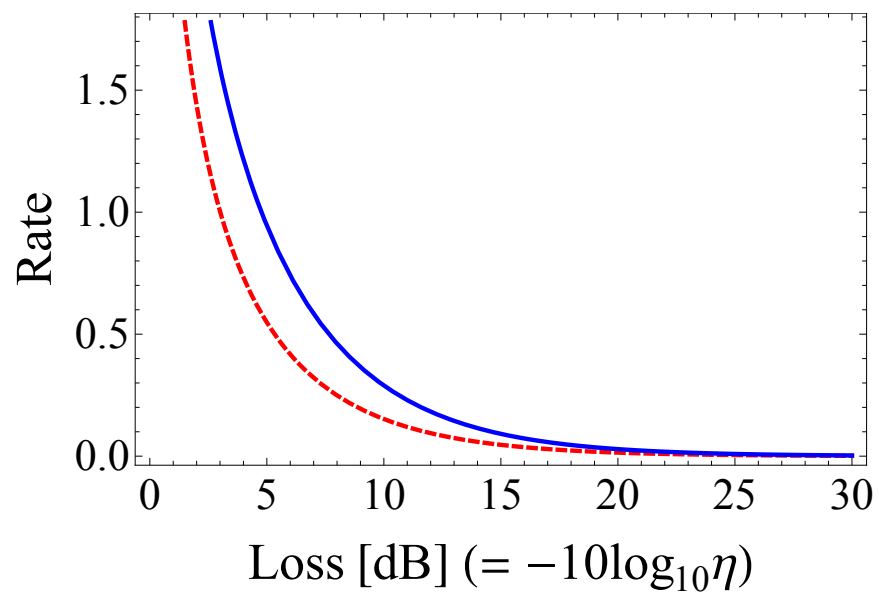

Figure 3: Upper and lower bounds on $Q_{2}$ and $P_{2}$ for a pure-loss bosonic channel. The dashed curve is both the direct and reverse coherent information, while the solid curve is the squashed entanglement upper bound.

Such channels have the following action on the mean vector $x$ and covariance matrix $\Gamma$ of a given single-mode, bosonic quantum state $\left.\mathrm{WPGP}^{+} 12\right]$ :

$$
\begin{aligned}
& x \rightarrow K x, \\
& \Gamma \rightarrow K \Gamma K^{T}+N,
\end{aligned}
$$

where $K$ and $N$ are square matrices satisfying

$$
\begin{aligned}
N & \geq 0, \\
\operatorname{det} N & \geq(\operatorname{det} K-1)^{2},
\end{aligned}
$$

in order for the map to be a legitimate completely positive and trace preserving map. A phaseinsensitive channel has

$$
\begin{aligned}
& K=\operatorname{diag}(\sqrt{\tau}, \sqrt{\tau}), \\
& N=\operatorname{diag}(\nu, \nu),
\end{aligned}
$$

where $\tau \in[0,1]$ corresponds to attenuation, $\tau \geq 1$ amplification, and $\nu$ is the variance of an additive noise.

A powerful (albeit simple) theorem in continuous-variable quantum information is that any phase-insensitive Gaussian channel as given above can be decomposed as the concatenation of a pure-loss channel $\mathcal{L}_{T}$ with loss parameter $T$ followed by an amplifier channel $\mathcal{A}_{G}$ with gain $G$, i.e.,

$$
\mathcal{N}=\mathcal{A}_{G} \circ \mathcal{L}_{T}
$$

where $\mathcal{N}$ is given by $(29)-(34)$ and $T$ and $G$ are chosen such that $\tau=T G$ and $\nu=G(1-T)+$ $G-1$ [CGH06, GPNBL ${ }^{+12}$. These equations are equivalent to $T=2 \tau /(\tau+\nu+1)$ and $G=$ $(\tau+\nu+1) / 2$. 
Now consider that $E_{\mathrm{sq}}(\mathcal{N})=E_{\mathrm{sq}}\left(\mathcal{A}_{G} \circ \mathcal{L}_{T}\right) \leq E_{\mathrm{sq}}\left(\mathcal{L}_{T}\right)$, where the inequality follows from quantum data processing (the quantum conditional mutual information does not increase under processing of one of the systems that is not the conditioning system-see the proof of Proposition 3 of [CW04]). Combining this fact with the bound for the pure-loss channel from the previous section, we find the following upper bounds on $Q_{2}(\mathcal{N})$ and $P_{2}(\mathcal{N})$ :

$$
Q_{2}(\mathcal{N}), P_{2}(\mathcal{N}) \leq \log _{2}\left(\frac{1+T}{1-T}\right)
$$

We can specialize the above result to the case of a thermal channel and an additive noise channel. The evolution for the thermal channel is the same as that in $(25)$, with the exception that the environment is prepared in a thermal state of mean photon number $N_{B} \geq 0$. The decomposition for the thermal channel then corresponds to that in (35), with

$$
\begin{aligned}
T & =\frac{\eta}{(1-\eta) N_{B}+1}, \\
G & =(1-\eta) N_{B}+1 .
\end{aligned}
$$

This is because for the thermal channel, we have $\tau=\eta$ and $\nu=(1-\eta)\left(2 N_{B}+1\right)$. Thus, we find the following upper bound on $Q_{2}$ and $P_{2}$ for the thermal channel:

$$
\log \left(\frac{1+\frac{\eta}{(1-\eta) N_{B}+1}}{1-\frac{\eta}{(1-\eta) N_{B}+1}}\right)=\log \left(\frac{(1-\eta) N_{B}+1+\eta}{(1-\eta) N_{B}+1-\eta}\right) .
$$

The additive noise channel corresponds to the following map:

$$
\rho \rightarrow \int d^{2} \alpha \frac{1}{\pi \bar{n}} \exp \left\{-|\alpha|^{2} / \bar{n}\right\} D(\alpha) \rho D^{\dagger}(\alpha)
$$

where $D(\alpha)$ is a unitary displacement operator and $\bar{n}>0$ is the noise variance $\left[\mathrm{WPGP}^{+} 12\right]$. It is well known that the additive noise channel is equivalent to a thermal channel for which $N_{B} \rightarrow \infty$ and $\eta \rightarrow 1$, while $(1-\eta) N_{B} \rightarrow \bar{n}\left[\mathrm{GGL}^{+} 04\right]$. From this relation, we immediately obtain the following upper bound on both $Q_{2}$ and $P_{2}$ for an additive noise channel:

$$
\log \left(\frac{\bar{n}+2}{\bar{n}}\right)
$$

\section{Conclusion}

We have established the squashed entanglement of a quantum channel as an operationally relevant, well behaved information measure for quantum channels. Our work here finds application in quantum key distribution, establishing the first simple upper bound on the rate that is achievable over the pure-loss bosonic channel, which models pure-loss free-space and fiber-optic communication. When the environment mode is noisy (for example, in a thermal state) - which is the case for a quantum key distribution setting for instance when the eavesdropper makes an active attack - the secret-key rate reduces from the case of the passive eavesdropper. In this case, the upper bound in (37) serves as a general upper bound to the rate achievable over a repeater-less lossy channel, using any key distribution protocol. 
An interesting open question is to establish $E_{\text {sq }}(\mathcal{N})$ as a strong converse rate for $Q_{2}(\mathcal{N})$ and $P_{2}(\mathcal{N})$ (Theorem 9 establishes $E_{\mathrm{sq}}(\mathcal{N})$ as a weak converse rate). A strong converse rate is defined to be such that if a communication scheme exceeds it, then the error probability converges to one as the number of channel uses becomes large. In this sense, establishing $E_{\mathrm{sq}}(\mathcal{N})$ as a strong converse rate for $Q_{2}(\mathcal{N})$ and $P_{2}(\mathcal{N})$ would significantly enhance Theorems 9 and 12 given here. For proving this, some combination of the ideas in BBCW13, Opp08 might be helpful.

Acknowledgements. We are grateful to Francesco Buscemi, Seth Lloyd, Cosmo Lupo, and Andreas Winter for insightful discussions. We also acknowledge Mark Byrd, Eric Chitambar, and the other participants of the Boris Musulin Workshop on Open Quantum Systems and Information Processing for helpful feedback. Finally, we thank Bob Tucci for kindly pointing us to his related work on squashed entanglement. This research was supported by the DARPA Quiness Program through US Army Research Office award W31P4Q-12-1-0019.

\section{A Squashed entanglement upper bound for the pure-loss bosonic channel}

Here we detail a proof that $(26)$ is an upper bound on $Q_{2}\left(\mathcal{N}_{\eta}\right)$ and $P_{2}\left(\mathcal{N}_{\eta}\right)$, where $\mathcal{N}_{\eta}$ is a pure-loss bosonic channel with transmissivity $\eta \in[0,1]$.

As mentioned before, we need to consider only pure states $|\phi\rangle_{A A^{\prime}}$ when optimizing the squashed entanglement of a quantum channel. Let $U_{E \rightarrow E^{\prime} F}^{\mathcal{S}}$ be an isometric extension of Eve's squashing channel $\mathcal{S}_{E \rightarrow E^{\prime}}$. Let $|\psi\rangle_{A B E^{\prime} F} \equiv U_{E \rightarrow E^{\prime} F}^{\mathcal{S}} U_{A^{\prime} \rightarrow B E}^{\mathcal{N}}|\phi\rangle_{A A^{\prime}}$, so that, $\operatorname{Tr}_{F}\left[|\phi\rangle\left\langle\left.\phi\right|_{A B E^{\prime} F}\right]=\mathcal{S}_{E \rightarrow E^{\prime}} \circ\right.$ $U_{A^{\prime} \rightarrow B E}^{\mathcal{N}}\left(\phi_{A A^{\prime}}\right)$. Then according to Lemma 4

$$
\sup _{\phi_{A A^{\prime}}} E_{\mathrm{Sq}}(A ; B)_{\mathcal{N}_{A^{\prime} \rightarrow B}\left(\phi_{A A^{\prime}}\right)}=\sup _{\phi_{A A^{\prime}}} \frac{1}{2} \inf _{\mathcal{S}_{E \rightarrow E^{\prime}}}\left(H\left(B \mid E^{\prime}\right)_{\psi}+H(B \mid F)_{\psi}\right) .
$$

Now suppose that Alice and Bob are connected by a pure-loss bosonic channel with transmissivity $\eta$. It is not necessarily an easy task to optimize Eve's squashing channel $\mathcal{S}$. Instead, we consider a specific squashing channel: a pure-loss bosonic channel $\mathcal{L}_{\eta_{1}}$ with transmissivity $\eta_{1}$. As shown in Lemma 4, the squashed entanglement can be written as a sum of two conditional entropies, each of which is a function of the reduced state $\operatorname{Tr}_{A}\left\{\phi_{A A^{\prime}}\right\}$ on $A^{\prime}$. Since the overall channel from $A^{\prime}$ to $B E^{\prime}$ is Gaussian and the overall channel from $A^{\prime}$ to $B F$ is Gaussian and due to the photon-number constraint at the input, it follows from the extremality of Gaussian states for conditional entropy EW07, WGC06 that a thermal state on $A^{\prime}$ of mean photon number $N_{S}$ maximizes both of these quantities. With this and the fact that $\phi_{A A^{\prime}}$ is a pure state, we can conclude that the optimal $\phi_{A A^{\prime}}$ is a two-mode squeezed vacuum (TMSV) state. Let $N_{S}$ be the average photon number of one share of the TMSV. Then the covariance matrix of the reduced thermal state at $A^{\prime}$ is given by

$$
\gamma^{A^{\prime}}=\left[\begin{array}{cc}
1+2 N_{S} & 0 \\
0 & 1+2 N_{S}
\end{array}\right]
$$

Note that the covariance matrix is defined such that a vacuum state (or coherent state) is described by an identity matrix. Therefore a covariance matrix of the initial state in system $A^{\prime} E^{\prime} F$ is given by $\gamma^{A^{\prime}} \oplus I^{E^{\prime}} \oplus I^{F}$. The beamsplitting operations are given by the transformation

$$
\gamma^{A^{\prime}} \oplus I^{E^{\prime}} \oplus I^{F} \rightarrow S_{\eta_{1}} S_{\eta}\left(\gamma^{A^{\prime}} \oplus I^{E^{\prime}} \oplus I^{F}\right) S_{\eta}^{T} S_{\eta_{1}}^{T},
$$


where

$$
S_{\eta}=\left[\begin{array}{ccc}
\sqrt{\eta} & \sqrt{1-\eta} & 0 \\
-\sqrt{1-\eta} & \sqrt{\eta} & 0 \\
0 & 0 & 1
\end{array}\right]^{\oplus 2}, \quad S_{\eta_{1}}=\left[\begin{array}{ccc}
1 & 0 & 0 \\
0 & \sqrt{\eta_{1}} & \sqrt{1-\eta_{1}} \\
0 & -\sqrt{1-\eta_{1}} & \sqrt{\eta_{1}}
\end{array}\right]^{\oplus 2},
$$

(the superscript " $\oplus 2$ " means that the same matrix is applied to both $x$ and $p$ quadratures. Because of the symmetry of the state and the beamsplitter operation in phase space, basically we need to consider only one quadrature.) This transformation is easily calculated and we get a covariance matrix for the state $\operatorname{Tr}_{A}\left\{|\phi\rangle\left\langle\left.\phi\right|_{A B E^{\prime} F}\right\}\right.$ :

$$
\begin{aligned}
S_{\eta_{1}} S_{\eta}\left(\gamma^{A^{\prime}} \oplus I^{E^{\prime}} \oplus I^{F}\right) S_{\eta_{1}}^{T} S_{\eta}^{T}= & \\
& {\left[\begin{array}{ccc}
1+\eta 2 N_{S} & -\sqrt{\eta(1-\eta)} \sqrt{\eta_{1}} 2 N_{S} & \sqrt{\eta(1-\eta)} \sqrt{1-\eta_{1}} 2 N_{S} \\
-\sqrt{\eta(1-\eta)} \sqrt{\eta_{1}} 2 N_{S} & 1+(1-\eta) \eta_{1} 2 N_{S} & -(1-\eta) \sqrt{\eta_{1}\left(1-\eta_{1}\right)} 2 N_{S} \\
\sqrt{\eta(1-\eta)} \sqrt{1-\eta_{1}} 2 N_{S} & -(1-\eta) \sqrt{\eta_{1}\left(1-\eta_{1}\right)} 2 N_{S} & 1+(1-\eta)\left(1-\eta_{1}\right) 2 N_{S}
\end{array}\right]^{\oplus 2} . }
\end{aligned}
$$

It immediately implies a covariance matrix of the marginal state on $E^{\prime}$ :

$$
\gamma_{E^{\prime}}=\left[\begin{array}{cc}
1+(1-\eta) \eta_{1} 2 N_{S} & 0 \\
0 & 1+(1-\eta) \eta_{1} 2 N_{S}
\end{array}\right]
$$

which is the covariance matrix for a thermal state with photon number $(1-\eta) \eta_{1} N_{S}$. Thus we have

$$
H\left(E^{\prime}\right)=g\left((1-\eta) \eta_{1} N_{S}\right)
$$

where $g(x)=(1+x) \log (1+x)-x \log x$. Similarly, we get

$$
H(F)=g\left((1-\eta)\left(1-\eta_{1}\right) N_{S}\right) .
$$

The other entropies $H\left(B E^{\prime}\right)$ and $H(B F)$ are also obtained by considering the corresponding submatrices and diagonalizing them. Then we can find

$$
\begin{aligned}
H\left(B E^{\prime}\right) & =g\left(\left\{\eta+(1-\eta) \eta_{1}\right\} N_{S}\right) \\
H(B F) & =g\left(\left\{\eta+(1-\eta)\left(1-\eta_{1}\right)\right\} N_{S}\right)
\end{aligned}
$$

As a consequence, we obtain the upper bound,

$$
\begin{aligned}
Q_{2}\left(\mathcal{N}_{\eta}\right) \leq & \min _{\eta_{1}} \frac{1}{2}\left\{g\left(\left\{\eta+(1-\eta) \eta_{1}\right\} N_{S}\right)-g\left((1-\eta) \eta_{1} N_{S}\right)\right. \\
& \left.+g\left(\left\{\eta+(1-\eta)\left(1-\eta_{1}\right)\right\} N_{S}\right)-g\left((1-\eta)\left(1-\eta_{1}\right) N_{S}\right)\right\} \\
= & g\left((1+\eta) N_{S} / 2\right)-g\left((1-\eta) N_{S} / 2\right) .
\end{aligned}
$$

The minimal value is achieved by $\eta_{1}=1 / 2$ because the function is symmetric and convex in $\eta_{1}$ (with convexity checked by computing the second derivative, see next appendix). The expression $g\left((1+\eta) N_{S} / 2\right)-g\left((1-\eta) N_{S} / 2\right)$ converges to $\log (1+\eta) /(1-\eta)$ as $N_{S} \rightarrow \infty$. 


\section{A.1 Convexity in $\eta_{1}$}

We compute the second derivative of the function in (41) in order to establish that it is convex. The function is

$$
g\left(\left(\eta+(1-\eta)\left(1-\eta_{1}\right)\right) N\right)+g\left(\left(\eta+(1-\eta) \eta_{1}\right) N\right)-g\left(\eta_{1}(1-\eta) N\right)-g\left(\left(1-\eta_{1}\right)(1-\eta) N\right)
$$

We now compute the second derivative of each term.

Consider that $g\left(\left(\eta+(1-\eta)\left(1-\eta_{1}\right)\right) N\right)$ is equal to

$$
\begin{aligned}
\left(\left(\eta+(1-\eta)\left(1-\eta_{1}\right)\right) N+1\right) \log ((\eta+ & \left.\left.(1-\eta)\left(1-\eta_{1}\right)\right) N+1\right) \\
& -\left(\left(\eta+(1-\eta)\left(1-\eta_{1}\right)\right) N\right) \log \left(\left(\eta+(1-\eta)\left(1-\eta_{1}\right)\right) N\right) .
\end{aligned}
$$

The first derivative of the above with respect to $\eta_{1}$ is given by

$$
\begin{aligned}
-(1-\eta) N \log ((\eta+ & \left.\left.(1-\eta)\left(1-\eta_{1}\right)\right) N+1\right)-(1-\eta) N \\
& \quad+(1-\eta) N \log \left(\left(\eta+(1-\eta)\left(1-\eta_{1}\right)\right) N\right)+(1-\eta) N \\
= & (1-\eta) N\left[-\log \left(\left(\eta+(1-\eta)\left(1-\eta_{1}\right)\right) N+1\right)+\log \left(\left(\eta+(1-\eta)\left(1-\eta_{1}\right)\right) N\right)\right]
\end{aligned}
$$

The second derivative with respect to $\eta_{1}$ is then given by

$$
\begin{aligned}
& (1-\eta) N\left[\frac{(1-\eta) N}{\left(\left(\eta+(1-\eta)\left(1-\eta_{1}\right)\right) N+1\right)}-\frac{(1-\eta) N}{\left(\left(\eta+(1-\eta)\left(1-\eta_{1}\right)\right) N\right)}\right] \\
& =-[(1-\eta) N]^{2}\left[\frac{1}{\left[\left(\eta+(1-\eta)\left(1-\eta_{1}\right)\right) N+1\right]\left[\left(\eta+(1-\eta)\left(1-\eta_{1}\right)\right) N\right]}\right] .
\end{aligned}
$$

Consider that $g\left(\left(\eta+(1-\eta) \eta_{1}\right) N\right)$ is equal to

$$
\left(\left(\eta+(1-\eta) \eta_{1}\right) N+1\right) \log \left(\left(\eta+(1-\eta) \eta_{1}\right) N+1\right)-\left(\left(\eta+(1-\eta) \eta_{1}\right) N\right) \log \left(\left(\eta+(1-\eta) \eta_{1}\right) N\right) .
$$

The first derivative of the above with respect to $\eta_{1}$ is given by

$$
\begin{aligned}
& (1-\eta) N \log \left(\left(\eta+(1-\eta) \eta_{1}\right) N+1\right)+(1-\eta) N \\
& -(1-\eta) N \log \left(\left(\eta+(1-\eta) \eta_{1}\right) N\right)-(1-\eta) N \\
& \quad=(1-\eta) N\left[\log \left(\left(\eta+(1-\eta) \eta_{1}\right) N+1\right)-\log \left(\left(\eta+(1-\eta) \eta_{1}\right) N\right)\right]
\end{aligned}
$$

The second derivative with respect to $\eta_{1}$ is then given by

$$
\begin{aligned}
& (1-\eta) N\left[\frac{(1-\eta) N}{\left(\left(\eta+(1-\eta) \eta_{1}\right) N+1\right)}-\frac{(1-\eta) N}{\left(\left(\eta+(1-\eta) \eta_{1}\right) N\right)}\right] \\
& =-[(1-\eta) N]^{2}\left[\frac{1}{\left[\left(\eta+(1-\eta) \eta_{1}\right) N+1\right]\left[\left(\eta+(1-\eta) \eta_{1}\right) N\right]}\right] .
\end{aligned}
$$

Consider that $g\left((1-\eta) \eta_{1} N\right)$ is equal to

$$
\left(\left((1-\eta) \eta_{1}\right) N+1\right) \log \left(\left((1-\eta) \eta_{1}\right) N+1\right)-\left(\left((1-\eta) \eta_{1}\right) N\right) \log \left(\left((1-\eta) \eta_{1}\right) N\right) .
$$


The first derivative of the above with respect to $\eta_{1}$ is given by

$$
\begin{aligned}
& (1-\eta) N \log \left(\left((1-\eta) \eta_{1}\right) N+1\right)+(1-\eta) N \\
& -(1-\eta) N \log \left(\left((1-\eta) \eta_{1}\right) N\right)-(1-\eta) N \\
& \quad=(1-\eta) N\left[\log \left(\left((1-\eta) \eta_{1}\right) N+1\right)-\log \left(\left((1-\eta) \eta_{1}\right) N\right)\right]
\end{aligned}
$$

The second derivative with respect to $\eta_{1}$ is then given by

$$
\begin{aligned}
& (1-\eta) N\left[\frac{(1-\eta) N}{\left(\left((1-\eta) \eta_{1}\right) N+1\right)}-\frac{(1-\eta) N}{\left(\left((1-\eta) \eta_{1}\right) N\right)}\right] \\
& =-[(1-\eta) N]^{2}\left[\frac{1}{\left[\left((1-\eta) \eta_{1}\right) N+1\right]\left[\left((1-\eta) \eta_{1}\right) N\right]}\right] .
\end{aligned}
$$

Consider that $g\left(\left((1-\eta)\left(1-\eta_{1}\right)\right) N\right)$ is equal to $\left(\left((1-\eta)\left(1-\eta_{1}\right)\right) N+1\right) \log \left(\left((1-\eta)\left(1-\eta_{1}\right)\right) N+1\right)-\left(\left((1-\eta)\left(1-\eta_{1}\right)\right) N\right) \log \left(\left((1-\eta)\left(1-\eta_{1}\right)\right) N\right)$.

The first derivative of the above with respect to $\eta_{1}$ is given by

$$
\begin{aligned}
-(1-\eta) N \log (((1-\eta) & \left.\left.\left(1-\eta_{1}\right)\right) N+1\right)-(1-\eta) N \\
+ & (1-\eta) N \log \left(\left((1-\eta)\left(1-\eta_{1}\right)\right) N\right)+(1-\eta) N \\
& =(1-\eta) N\left[-\log \left(\left((1-\eta)\left(1-\eta_{1}\right)\right) N+1\right)+\log \left(\left((1-\eta)\left(1-\eta_{1}\right)\right) N\right)\right]
\end{aligned}
$$

The second derivative with respect to $\eta_{1}$ is then given by

$$
\begin{aligned}
& (1-\eta) N\left[\frac{(1-\eta) N}{\left(\left((1-\eta)\left(1-\eta_{1}\right)\right) N+1\right)}-\frac{(1-\eta) N}{\left(\left((1-\eta)\left(1-\eta_{1}\right)\right) N\right)}\right] \\
& =-[(1-\eta) N]^{2}\left[\frac{1}{\left[\left((1-\eta)\left(1-\eta_{1}\right)\right) N+1\right]\left[\left((1-\eta)\left(1-\eta_{1}\right)\right) N\right]}\right] .
\end{aligned}
$$

So now we just need to determine whether the second derivative is positive:

$$
\begin{aligned}
& -[(1-\eta) N]^{2}\left[\frac{1}{\left[\left(\eta+(1-\eta)\left(1-\eta_{1}\right)\right) N+1\right]\left[\left(\eta+(1-\eta)\left(1-\eta_{1}\right)\right) N\right]}\right] \\
& -[(1-\eta) N]^{2}\left[\frac{1}{\left[\left(\eta+(1-\eta) \eta_{1}\right) N+1\right]\left[\left(\eta+(1-\eta) \eta_{1}\right) N\right]}\right] \\
& +[(1-\eta) N]^{2}\left[\frac{1}{\left[\left((1-\eta) \eta_{1}\right) N+1\right]\left[\left((1-\eta) \eta_{1}\right) N\right]}\right] \\
& +[(1-\eta) N]^{2}\left[\frac{1}{\left[\left((1-\eta)\left(1-\eta_{1}\right)\right) N+1\right]\left[\left((1-\eta)\left(1-\eta_{1}\right)\right) N\right]}\right] .
\end{aligned}
$$

This simplifies to

$$
\begin{aligned}
& {\left[\frac{1}{\left[\left((1-\eta) \eta_{1}\right) N+1\right]\left[\left((1-\eta) \eta_{1}\right) N\right]}\right]+} \\
& {\left[\frac{1}{\left[\left((1-\eta)\left(1-\eta_{1}\right)\right) N+1\right]\left[\left((1-\eta)\left(1-\eta_{1}\right)\right) N\right]}\right]} \\
& \geq\left[\frac{1}{\left[\left(\eta+(1-\eta) \eta_{1}\right) N+1\right]\left[\left(\eta+(1-\eta) \eta_{1}\right) N\right]}\right]+ \\
& {\left[\frac{1}{\left[\left(\eta+(1-\eta)\left(1-\eta_{1}\right)\right) N+1\right]\left[\left(\eta+(1-\eta)\left(1-\eta_{1}\right)\right) N\right]}\right]}
\end{aligned}
$$


This last inequality is true by inspection because the terms on the RHS are the same as those on the LHS, only with an extra factor of $\eta \geq 0$ in the denominator.

\section{References}

[AC93] Rudolf Ahlswede and Imre Csiszár. Common randomness in information theory and cryptography. I. Secret sharing. IEEE Transactions on Information Theory, 39(4):1121-1132, July 1993.

[AF04] Robert Alicki and Mark Fannes. Continuity of quantum conditional information. Journal of Physics A: Mathematical and General, 37(5):L55-L57, 2004. arXiv:quant$\mathrm{ph} / 0312081$.

[BBCW13] Mario Berta, Fernando G. S. L. Brandão, Matthias Christandl, and Stephanie Wehner. Entanglement cost of quantum channels. IEEE Transactions on Information Theory, 59(10):6779-6795, October 2013. arXiv:1108.5357.

[BCY11] Fernando G. S. L. Brandão, Matthias Christandl, and Jon Yard. Faithful squashed entanglement. Communications in Mathematical Physics, 306(3):805-830, September 2011. arXiv:1010.1750.

[BDS97] Charles H. Bennett, David P. DiVincenzo, and John A. Smolin. Capacities of quantum erasure channels. Physical Review Letters, 78(16):3217-3220, April 1997. arXiv:quant-ph/9701015.

[CEH $\left.{ }^{+} 07\right]$ Matthias Christandl, Artur Ekert, Michal Horodecki, Pawel Horodecki, Jonathan Oppenheim, and Renato Renner. Unifying classical and quantum key distillation. Proceedings of the 4 th Theory of Cryptography Conference, Lecture Notes in Computer Science, 4392:456-478, February 2007. arXiv:quant-ph/0608199.

[CGH06] Filippo Caruso, Vittorio Giovannetti, and Alexander S. Holevo. One-mode bosonic Gaussian channels: a full weak-degradability classification. New Journal of Physics, 8(12):310, 2006. arXiv:quant-ph/0609013.

[Chr02] Matthias Christandl. The quantum analog to intrinsic information. Diploma Thesis, ETH Zurich, unpublished, 2002.

[Chr06] Matthias Christandl. The Structure of Bipartite Quantum States: Insights from Group Theory and Cryptography. PhD thesis, University of Cambridge, April 2006. arXiv:quant-ph/0604183.

[CRW03] Matthias Christandl, Renato Renner, and Stefan Wolf. A property of the intrinsic mutual information. In Proceedings of the International Symposium on Information Theory, page 258, Yokohama, Japan, June 2003.

[CW04] Matthias Christandl and Andreas Winter. "Squashed entanglement": An additive entanglement measure. Journal of Mathematical Physics, 45(3):829-840, March 2004. arXiv:quant-ph/0308088. 
[EW07] Jens Eisert and Michael M. Wolf. Quantum Information with Continous Variables of Atoms and Light, chapter Gaussian quantum channels, pages 23-42. Imperial College Press, 2007. arXiv:quant-ph/0505151.

$\left[\mathrm{GGL}^{+} 04\right] \quad$ Vittorio Giovannetti, Saikat Guha, Seth Lloyd, Lorenzo Maccone, and Jeffrey H. Shapiro. Minimum output entropy of bosonic channels: A conjecture. Physical Review A, 70:032315, September 2004. arXiv:quant-ph/0404005.

[GPLS09] Raúl García-Patrón, Stefano Pirandola, Seth Lloyd, and Jeffrey H. Shapiro. Reverse coherent information. Physical Review Letters, 102:210501, May 2009. arXiv:0808.0210.

[GPNBL $\left.{ }^{+} 12\right]$ Raul Garcia-Patron, Carlos Navarrete-Benlloch, Seth Lloyd, Jeffrey H. Shapiro, and Nicolas J. Cerf. Majorization theory approach to the Gaussian channel minimum entropy conjecture. Physical Review Letters, 108:110505, March 2012. arXiv:1111.1986.

[HHHO05] Karol Horodecki, Michał Horodecki, Paweł Horodecki, and Jonathan Oppenheim. Secure key from bound entanglement. Physical Review Letters, 94(16):160502, April 2005. arXiv:quant-ph/0309110.

[HHHO09] Karol Horodecki, Michal Horodecki, Pawel Horodecki, and Jonathan Oppenheim. General paradigm for distilling classical key from quantum states. IEEE Transactions on Information Theory, 55(4):1898-1929, April 2009. arXiv:quant-ph/0506189.

[HW01] Alexander S. Holevo and Reinhard F. Werner. Evaluating capacities of bosonic Gaussian channels. Physical Review A, 63:032312, February 2001.

[KW04] Masato Koashi and Andreas Winter. Monogamy of quantum entanglement and other correlations. Physical Review A, 69:022309, February 2004. arXiv:quant-ph/0310037.

[LC99] Hoi-Kwong Lo and Hoi Fung Chau. Unconditional security of quantum key distribution over arbitrarily long distances. Science, 283(5410):2050-2056, 1999. arXiv:quant-ph/9803006.

[LR73] Elliott H. Lieb and Mary Beth Ruskai. Proof of the strong subadditivity of quantummechanical entropy. Journal of Mathematical Physics, 14:1938-1941, 1973.

[Mau93] Ueli M. Maurer. Secret key agreement by public discussion from common information. IEEE Transactions on Information Theory, 39(3):733-742, May 1993.

[MW99] Ueli M. Maurer and Stefan Wolf. Unconditionally secure key agreement and the intrinsic conditional information. IEEE Transactions on Information Theory, 45(2):499-514, March 1999.

[Opp08] Jonathan Oppenheim. A paradigm for entanglement theory based on quantum communication. January 2008. arXiv:0801.0458.

[PGBL09] Stefano Pirandola, Raul García-Patrón, Samuel L. Braunstein, and Seth Lloyd. Direct and reverse secret-key capacities of a quantum channel. Physical Review Letters, 102:050503, February 2009. arXiv:0809.3273. 
[Rai01] Eric M. Rains. A semidefinite program for distillable entanglement. IEEE Transactions on Information Theory, 47(7):2921-2933, November 2001. arXiv:quant$\mathrm{ph} / 0008047$.

[SP00] Peter W. Shor and John Preskill. Simple proof of security of the BB84 quantum key distribution protocol. Physical Review Letters, 85(2):441, 2000. arXiv:quant$\mathrm{ph} / 0003004$.

[SW98] Benjamin Schumacher and Michael D. Westmoreland. Quantum privacy and quantum coherence. Physical Review Letters, 80(25):5695-5697, June 1998. arXiv:quant$\mathrm{ph} / 9709058$.

[Tuc99] Robert R. Tucci. Quantum entanglement and conditional information transmission. 1999. arXiv:quant-ph/9909041.

[Tuc02] Robert R. Tucci. Entanglement of distillation and conditional mutual information. 2002. arXiv:quant-ph/0202144.

[Uhl77] Armin Uhlmann. Relative entropy and the Wigner-Yanase-Dyson-Lieb concavity in an interpolation theory. Communications in Mathematical Physics, 54:21-32, 1977.

[WGC06] Michael M. Wolf, Geza Giedke, and J. Ignacio Cirac. Extremality of Gaussian quantum states. Physical Review Letters, 96:080502, March 2006. arXiv:quant$\mathrm{ph} / 0509154$.

[WPGG07] Michael M. Wolf, David Pérez-García, and Geza Giedke. Quantum capacities of bosonic channels. Physical Review Letters, 98(13):130501, March 2007.

[WPGP $\left.^{+} 12\right]$ Christian Weedbrook, Stefano Pirandola, Raul Garcia-Patron, Nicolas J. Cerf, Timothy C. Ralph, Jeffrey H. Shapiro, and Seth Lloyd. Gaussian quantum information. Reviews of Modern Physics, 84:621-669, May 2012. arXiv:1110.3234. 